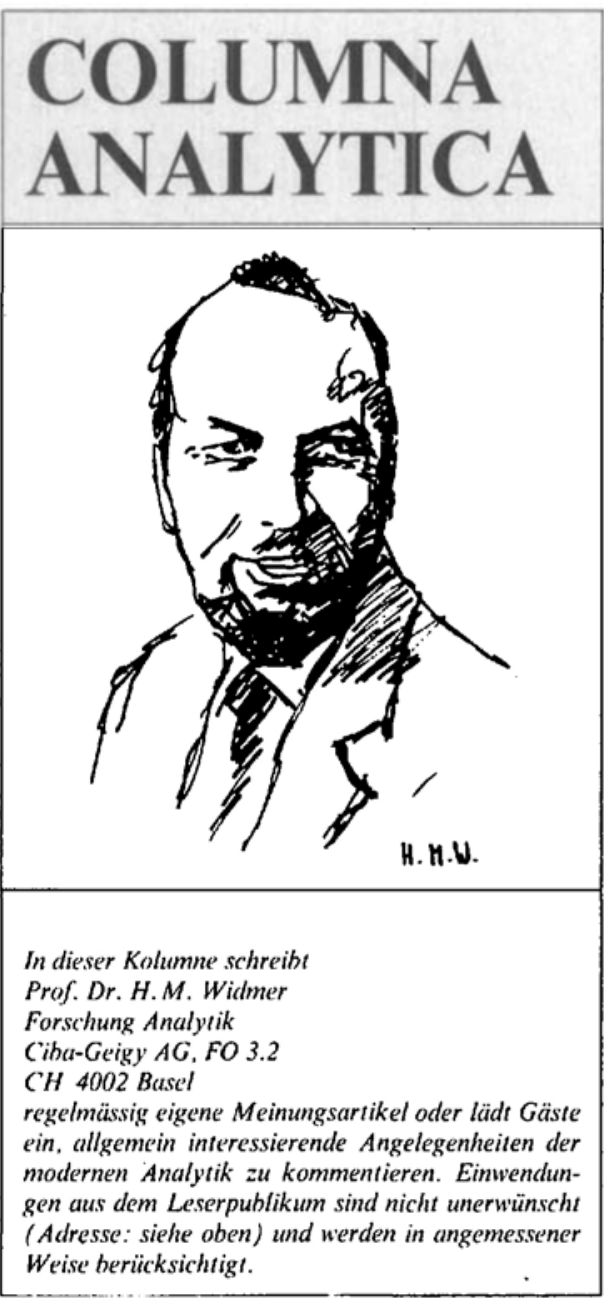

Das schweizerische Brutto-Sozialprodukt der letzten Jahre lag bei ungefähr 220 Milliarden Franken, wobei die Metallindustrie rund $25 \%$ (55 Md. Fr.), der Handel gut $17 \%$ (40 Md. Fr.), das Bankwesen $10 \%$ (23 Md. Fr.), das Baugewerbe $8 \%$ (17 Md. Fr.) und die Landwirtschaft $4 \%(8$ Md. Fr.) beisteuerten.

Bei diesen ausgewiesenen Grössen ist es interessant abzuschätzen, wie gross der jährliche Aufwand für chemisch-analytische Untersuchungen in der Schweiz ist. Wir schätzen diesen auf mehrere Milliarden Franken pro Jahr, wobei allein in der schweizerischen chemischen Industrie für analytische Aufgaben annähernd eine Milliarde Franken an realen Kosten ausgegeben wird. Zählt man die analytischen Aufwendungen der übrigen Industrien (z.B. Nahrungsmittelsektor), der Hochschulen, kantonalen und eidgenössischen Einrichtungen und diejenigen der medizinischen und klinischen Anstalten sowie der wissenschaftlichen, medizinischen und technischen Forschung, aber auch diejenigen des Umweltschutzes, der Toxikologie und Arbeitshygiene und anderer Zweige der angewandten Wissenschaften (z. B. Forensik), Kultur (z.B. Archäologie) und Künste (Konservierung von Kunstgegenständen und deren Echtheitsprüfung) dazu, so ergibt sich eine Multimilliarden-FrankenSumme, die sich durchaus mit dem Beitrag der Landwirtschaft am Brutto-Sozialprodukt der Schweiz vergleichen lässt.
Chimia 44 (1990) 298-299

(C) Schweiz. Chemiker-Verband; ISSN $0009-4293$

\title{
'Comité Suisse de Chimie Analytique' (CSCA)
}

Für analytische Instrumente, vorwiegend der Chromatographie und Spektrometrie, werden in der Schweiz jährlich mehr als 30 Millionen Franken ausgegeben und fast das Doppelte wird für die chromatographische und spektrometrische Infrastruktur (Säulenmaterialien, Chemikalien, Lösungsmittel, allgemeines Verbrauchsmaterial) und die dazugehörige Software aufgewendet.

Durch die Fortschritte der Instrumentalanalytik sind in den letzten dreissig Jahren neue Arbeitsgebiete für die analytischen Untersuchungen erschlossen worden und der Wirkungskreis analytisch-chemischer Methoden beginnt, die Biologie, Medizin und die klinischen Belange (Biopolymere) zu erobern. Mit den gleichen Technologien, die in der analytischen Chemie eingesetzt werden, lassen sich nämlich heute schon Bedürfnisse der Biotechnologie und Molekularbiologie abdecken. Es war Barry L. Karger (Leiter des Barnett-Institutes an der Northeastern University in Boston), der vor wenigen Jahren die Begriffe 'Analytical Biotechnology' und 'Analytical Molecular Biology' schuf und auf die wachsende Bedeutung dieser Disziplinen hinwies. Damit sprengt die Analytik als Wissenschaft den ihr angestammten Rahmen der Chemie, in dem sie bisher seit fast 400 Jahren eingespannt war.

Die analytische Chemie, und in Zukunft auch die analytische Biotechnologie und analytische Molekularbiologie, stellen damit in der Schweiz einen ernstzunehmenden Wirtschaftssektor dar. In der Tat liessen sich bei einer systematischen Ausnutzung der Möglichkeiten der sich dynamisch entwickelnden und erneuernden Instrumentalanalytik bedeutende wirtschaftliche Vorteile erarbeiten, wenn die Neuerungen voll ausgenutzt werden könnten. Wir haben kürzlich gezeigt, dass die Instrumentalanalytik durch eine Steigerung der Leistungsfähigkeit im Ausmass von einer Grössenordnung pro 10 Jahre charakterisiert ist (Chimia 1990, 44, 63), was einer jährlichen Verbesserung um mehr als $25 \%$ entspricht.

Natürlich kann dieses Potential nie voll ausgeschöpft werden, weil die Instrumente über Jahre hinaus amortisiert werden müssen. Wir beschreiben aber trotzdem ein Beispiel zur Illustration dieser Möglichkeiten und wollen die relative Leistungsfähigkeit von zwei analytischen Laboratorien vergleichen, die sich durch die gleichen Alltagsuntersuchungen auszeichnen, somit die gleichen Methoden einsetzen, aber diese Aufgaben mit einem unterschiedlichen Instrumentenpark angehen; im einen mit etwa 5 bis 7 Jahre alten, manuell zu bedienenden Geräten (z. B. Flüssigchromatographen), im andern mit der neusten voll automatisierten Instrumentengeneration, d.h. höchstens 3 Jahre alten Instrumenten. Es ist ohne weiteres einzusehen, dass mit den ältern, nicht automatisierten Instrumenten im Tag nur ein Drittel bis die Hälfte der Analysen ausgeführt werden können, die im 24-Stunden-Betrieb mit den neueren Geräten erledigt werden. Es versteht sich auch, dass damit der Preis der Einzelanalyse drastisch fällt, die moderneren Instrumente billigere Analysen ermöglichen.

Spielt der Zeitfaktor eine wesentliche Rolle zur Steigerung der Produktivität, dann ist es auch wichtig, traditionelle, zeitaufwendigere Methoden durch neuere, schnellere zu ersetzen. Auch hier möchten wir ein Beispiel zur Illustration anbringen. Die Erfahrung in unseren Laboratorien zeigt, dass, wo immer die Möglichkeit besteht, die Flüssigchromatographie (LC) durch 'Supercritical Fluid Chromatography' (SFC) zu ersetzen, letztere um einen Faktor 3 bis 6 raschere Analysen erlaubt, was zudem vom Umstand begleitet ist, dass auch die Methodenentwicklung und Probenvorbereitung in der SFC im allgemeinen weniger anspruchsvoll und weniger zeitaufwendig ist.

Um solche Effekte wirtschaftlich auszunutzen und sie im industriellen Konkurrenzkampf erfolgreich in die Waagschale werfen zu können, braucht es viel Spürsinn, Erfahrung und technisches Verständnis. Es gilt, diese durch Ausbildung, Praxis und durch Weiterbildung zu fördern, und es braucht dazu das nötige Verständnis für die Möglichkeiten und Bedürfnisse der modernen analytischen Chemie.

Die analytischen Fortschritte, die mit der kaum ein halbes Jahrhundert alten Instrumentalanalytik eine nie zuvor beobachtete Dynamik erreichte, ist aber gerade infolge dieser Dynamik ins Zielfeld von Missverständnissen, Vorurteilen und falscher Einschätzung geraten. Die moderne Analytik muss sich durch Öffentlichkeitsarbeit, Promotion und publik gemachte Erfolge das nötige Gehör verschaffen, damit ihr vom Industriemanagement, den Bildungsverantwortlichen, den amtlichen Stellen und nicht zuletzt auch den Politikern die ihrem Potential entsprechende Be- 
deutung zugesprochen wird und sie im geeigneten Umfeld ihre Stärke ausspielen und ihre wahre wirtschaftliche Bedeutung unter Beweis stellen kann.

Kein Land wie die Schweiz weist ein derart hohes analytisches Know-how pro Kapita auf, und doch stimmen die Voraussetzungen für eine wahrhaftig blühende Analytik nicht. An den Hochschulen fristet die analytische Forschung und Ausbildung mit wenigen Ausnahmen immer noch ein Schattendasein, und die Schweiz hat es auch nicht verstanden, ihr analytisches 'Know-how' in Gestalt der Instrumentalanalytik zu vermarkten, obwohl gerade mit dem Standort Schweiz die denkbar besten Voraussetzungen anzutreffen waren und immer noch anzutreffen sind, so zum Beispiel auf den Neuigkeitsgebieten der chemischen Sensoren und Biosensoren und der oben erwähnten analytischen Biotechnologie und analytischen Molekularbiologie, die für die immer wichtiger werdende medizinische Diagnostik und klinischen Untersuchungen von beachtlicher wirtschaftlicher Bedeutung sein könnte.

Im Lichte der bevorstehenden Fusion zwischen der Schweizerischen Chemischen Gesellschaft und dem Schweizerischen Chemiker-Verband und aufgrund einer durch solche Gedanken ausgelösten Analyse, die von einer aus einem halben Dutzend namhafter Schweizer Analytiker zusammengesetzten Gruppe am 15. November 1989 in Bern vorgenommen wurde, gründete diese Versammlung ein Interes- senkomitee, das Schweizerische Komitee für Analytische Chemie (Comité Suisse de Chimie Analytique, CSCA). In der Zwischenzeit fanden zwei weitere Sitzungen des CSCA statt, wobei der Teilnehmerkreis wesentlich erweitert wurde und den Worten auch Taten folgten.

Im CSCA sind die Hochschulen, eidgenössischen Anstalten und die chemische Industrie repräsentativ vertreten. Auch die einzelnen analytischen Interessengruppen und professionellen Vereinigungen sind durch ihre Präsidenten bestens vertreten. Es wurde versucht, andere Industriezweige, die klinische, medizinische Chemie und Lebensmittelchemie zu berücksichtigen, und es wurde Gewicht darauf gelegt, dass die französisch sprechende Schweiz wie die Deutschschweiz vertreten ist. Noch ist es bis jetzt nicht gelungen, im CSCA einen Vertreter aus der italienischen Schweiz engagieren zu können.

Das CSCA will sich für die Förderung der analytischen Chemie im weitesten Sinne, die Bildung und Weiterbildung auf dem Gebiet der analytischen Chemie einsetzen und in diesem Sinne aktiv werden. Es geht aber auch darum, im Rahmen der interdisziplinären Natur der modernen Analytik entsprechende Öffentlichkeitsarbeit zu leisten, gemeinsame Ziele der verschiedenen analytischen Disziplinen zu finden und diese gemeinsam anzupeilen.

Bereits anlässlich der Gründungssitzung beschloss das CSCA, der Schweizerischen Chemischen Gesellschaft das Gesuch zu stellen, an der Herbstversammlung vom 19. Oktober 1990 in Bern ein ganztägiges Analytikseminar durchführen zu dürfen. Diesem Antrag wurde entsprochen und durch ein Dutzend Vorträge und über dreissig Posters wird es gelingen, ein repräsentatives Schaufenster der schweizerischen Analytischen Chemie vorzuführen. Dabei kommen auf Einladung des CSCA auch zwei namhafte, international bekannte Analytiker zu Wort. Professor Csaba Horvath von der Yale University in New Haven, Connecticut, ein Pionier der LC, wird über die Möglichkeiten der ultraschnellen LC referieren, während Professor Shigeru Terabe vom Himeji Institute of Technology, Shosha, Himeji, Hyogo in Japan, bekannt für seine Arbeiten auf dem Gebiet der 'Micellar Electrokinetic Chromatography' (MECC), über neuste Entwicklungen auf diesem Spezialgebiet der Kapillarelektrophorese mit Micellen berichten wird.

Mit dem CSCA, einem reinen Interessenkomitee ohne Vereinsstatut, wird versucht, der modernen Analytik vermehrt Bedeutung zu verhelfen und der schweizerischen 'High Tech'-Analytik unter Umständen auch eine verbesserte wirtschaftliche Grundlage zu verschaffen. Das CSCA ist gewillt, die Zeitschrift CHIMIA vermehrt als Medium zur Exposition solcher Leistungen zu benutzen und diese als Sprachrohr zur Kommunikation von analytischen Bedürfnissen zu gebrauchen.

\section{Schweizerisches Komitee für Chemie Comité Suisse de la Chimie (CSC)}

Das Comité Suisse de la Chimie (CSC) ist die Dachorganisation von Chemischen Vereinigungen in der Schweiz. In dieser Funktion übernimmt es vor allem Aufgaben, die den Rahmen der einzelnen Mitgliedgesellschaften übersteigen oder von allgemeinem Interesse für die Chemie sind (gesamtschweizerische Koordinationsaufgaben, insbesondere auf den Gebieten Offentlichkeitsarbeit und Chemieunterricht; Landesvertretungen bei internationalen Organisationen).

ILMAC' '90:

Informationsveranstaltung

\section{Dienstag, 23. Oktober 1990, 15.00 Uhr Kongresszentrum der Schweizer Mustermesse Basel}

Nach den grossen Erfolgen in den Jahren 1984 und 1987 - jewcils an dic 1000 Teilnehmer, die meisten aus der Nordwestschweiz - organisiert das CSC auch heuer Vorträge, die sich wicderum zur Hauptsache an Schüler und Schülerinnen der Oberstufe von Mittelschulen und an die Lehrerschaft (Chemie, Biologie) richten. Eine Programmkommission aus Vertretern von Gymnasien, Hochschulen, Industrie und Presse hat sich für ein aktuelles Thema aus den Domänen Chemie - Biologie ... Physiologie - Verhaltensforschung entschieden. Es sollte nicht nur junge Menschen faszinieren und ihre Interessen wecken, sondern auch weiteren Teilnehmern z. B. Chemikern, Biologen - wertvolle Informationen vermittein.
Der Eintritt zu den Vorträgen, die von Dias, Filmen und Videos begleitet werden, ist für alle Interessenten frei. Eine dem Thema gewidmete Sondernummer der Zeitschrift für Naturwissenschaftslehrer 'Chemie und Biologie' wird vor dem Vortragsreferat aufliegen. Falls es die Zeit erlaubt, sollen auch Fragen an die Referenten gestellt werden können.

\section{Programm}

Chemische Signale und ihre Empfänger

Tagungsleiter: Prof. W. Simon, Laboratorium für Organische Chemie der ETH Zürich

Begrüssung und Einführung durch den Tagungsleiter

Dr. U. Burckhardt, CIBA-GEIGY AG, Basel Die Sprache der Kirschenfliege

Prof. H.J. Bestmann, Universität Erlangen, BRD Chemische Informationsübermittlung bei Insekten

Dozenten, Assistenten (Universitäten, ETH, HTL) die eine Gruppe (Doktoranden, Studenten) formieren können, erhalten Gratiskarten für den Besuch der Fachmesse ILMAC '90 (23.-26. Oktober 1990) an einem beliebigen Tag. Aus organisatorischen Gründen ist dafür allerdings eine schriftliche Anmeldung zur Teilnahme an die folgende Adresse bis zum 14. Oklober 1990 unter Angabe der Teilnehmerzahl und einer Empfangsadresse für den Versand erforderlich:

Für das CSC: Dr. A. Fürst, KfO

F. Hoffmann-La Roche AG, 71/113 Postfach

4002 Basel
ILMAC

11. Internationale

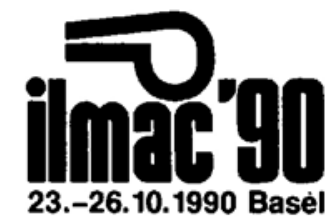

Chemie-Fachmesse

Ein Treffpunkt von Technik und Wissenschaft

Veranstaltet vom Schweizerischen Chemikerverband (SChV) findet die ILMAC - Internationale Chemie-Fachmesse - vom 23. bis 26 . Oktober' 1990 bereits zum 11. Mal in Basel im Rahmen der Schweizer Mustermesse statt. Thre Schwerpunkte sind Laborato riums- und Verfahrenstechnik, Messtechnik und Automatik. Die Fachmesse wird begleitet vom ILMACKongress. Beide Veranstaltungen stehen unter den Generalthema 'Neue Werkstoffe - neue Technologien'.

Uber 450 Aussteller werden an der ILMAC ihre Produkte und Dienstleistungen präsentieren. $\mathrm{Zu}$ den Ausstellungs-Schwerpunkten gehören erstmals auch Laboreinrichtungen für Arztpraxen. Weiter zählen dazu Biotechnologie, Isotopen und Radiochemie, Verfahrens- und Automationstechnik, Laborausstattung und -messtechnik sowie Sicherheit, Umweltschutz, Ausbildung und Institutionen. Die ILMAC gehört zu den international bedeutendsten europäischen Chemicfachmessen und findet alle drei Jahre statt. Sic schafft das Forum, den Markt, auf dem Innovationen dem Praktiker vorgestellt werden, auf dem der Forscher seine Bedürfnisse nach Ausrüstungen und Messgeräten anbringen und unter dem vielfältigen Angebot auswählen kann.

Eine Sonderschau steht unter dem Titel Explosionsschutz und wird gemeinsam vom Schweizerischen Ver- 
deutung zugesprochen wird und sie im geeigneten Umfeld ihre Stärke ausspielen und ihre wahre wirtschaftliche Bedeutung unter Beweis stellen kann.

Kein Land wie die Schweiz weist ein derart hohes analytisches Know-how pro Kapita auf, und doch stimmen die Voraussetzungen für eine wahrhaftig blühende Analytik nicht. An den Hochschulen fristet die analytische Forschung und Ausbildung mit wenigen Ausnahmen immer noch ein Schattendasein, und die Schweiz hat es auch nicht verstanden, ihr analytisches 'Know-how' in Gestalt der Instrumentalanalytik zu vermarkten, obwohl gerade mit dem Standort Schweiz die denkbar besten Voraussetzungen anzutreffen waren und immer noch anzutreffen sind, so zum Beispiel auf den Neuigkeitsgebieten der chemischen Sensoren und Biosensoren und der oben erwähnten analytischen Biotechnologie und analytischen Molekularbiologie, die für die immer wichtiger werdende medizinische Diagnostik und klinischen Untersuchungen von beachtlicher wirtschaftlicher Bedeutung sein könnte.

Im Lichte der bevorstehenden Fusion zwischen der Schweizerischen Chemischen Gesellschaft und dem Schweizerischen Chemiker-Verband und aufgrund einer durch solche Gedanken ausgelösten Analyse, die von einer aus einem halben Dutzend namhafter Schweizer Analytiker zusammengesetzten Gruppe am 15. November 1989 in Bern vorgenommen wurde, gründete diese Versammlung ein Interes- senkomitee, das Schweizerische Komitee für Analytische Chemie (Comité Suisse de Chimie Analytique, CSCA). In der Zwischenzeit fanden zwei weitere Sitzungen des CSCA statt, wobei der Teilnehmerkreis wesentlich erweitert wurde und den Worten auch Taten folgten.

Im CSCA sind die Hochschulen, eidgenössischen Anstalten und die chemische Industrie repräsentativ vertreten. Auch die einzelnen analytischen Interessengruppen und professionellen Vereinigungen sind durch ihre Präsidenten bestens vertreten. Es wurde versucht, andere Industriezweige, die klinische, medizinische Chemie und Lebensmittelchemie zu berücksichtigen, und es wurde Gewicht darauf gelegt, dass die französisch sprechende Schweiz wie die Deutschschweiz vertreten ist. Noch ist es bis jetzt nicht gelungen, im CSCA einen Vertreter aus der italienischen Schweiz engagieren zu können.

Das CSCA will sich für die Förderung der analytischen Chemie im weitesten Sinne, die Bildung und Weiterbildung auf dem Gebiet der analytischen Chemie einsetzen und in diesem Sinne aktiv werden. Es geht aber auch darum, im Rahmen der interdisziplinären Natur der modernen Analytik entsprechende Öffentlichkeitsarbeit zu leisten, gemeinsame Ziele der verschiedenen analytischen Disziplinen zu finden und diese gemeinsam anzupeilen.

Bereits anlässlich der Gründungssitzung beschloss das CSCA, der Schweizerischen Chemischen Gesellschaft das Gesuch zu stellen, an der Herbstversammlung vom 19. Oktober 1990 in Bern ein ganztägiges Analytikseminar durchführen zu dürfen. Diesem Antrag wurde entsprochen und durch ein Dutzend Vorträge und über dreissig Posters wird es gelingen, ein repräsentatives Schaufenster der schweizerischen Analytischen Chemie vorzuführen. Dabei kommen auf Einladung des CSCA auch zwei namhafte, international bekannte Analytiker zu Wort. Professor Csaba Horvath von der Yale University in New Haven, Connecticut, ein Pionier der LC, wird über die Möglichkeiten der ultraschnellen LC referieren, während Professor Shigeru Terabe vom Himeji Institute of Technology, Shosha, Himeji, Hyogo in Japan, bekannt für seine Arbeiten auf dem Gebiet der 'Micellar Electrokinetic Chromatography' (MECC), über neuste Entwicklungen auf diesem Spezialgebiet der Kapillarelektrophorese mit Micellen berichten wird.

Mit dem CSCA, einem reinen Interessenkomitee ohne Vereinsstatut, wird versucht, der modernen Analytik vermehrt Bedeutung zu verhelfen und der schweizerischen 'High Tech'-Analytik unter Umständen auch eine verbesserte wirtschaftliche Grundlage zu verschaffen. Das CSCA ist gewillt, die Zeitschrift CHIMIA vermehrt als Medium zur Exposition solcher Leistungen zu benutzen und diese als Sprachrohr zur Kommunikation von analytischen Bedürfnissen zu gebrauchen.

\section{Schweizerisches Komitee für Chemie Comité Suisse de la Chimie (CSC)}

Das Comité Suisse de la Chimie (CSC) ist die Dachorganisation von Chemischen Vereinigungen in der Schweiz. In dieser Funktion übernimmt es vor allem Aufgaben, die den Rahmen der einzelnen Mitgliedgesellschaften übersteigen oder von allgemeinem Interesse für die Chemie sind (gesamtschweizerische Koordinationsaufgaben, insbesondere auf den Gebieten Offentlichkeitsarbeit und Chemieunterricht; Landesvertretungen bei internationalen Organisationen).

ILMAC' '90:

Informationsveranstaltung

\section{Dienstag, 23. Oktober 1990, 15.00 Uhr Kongresszentrum der Schweizer Mustermesse Basel}

Nach den grossen Erfolgen in den Jahren 1984 und 1987 - jewcils an dic 1000 Teilnehmer, die meisten aus der Nordwestschweiz - organisiert das CSC auch heuer Vorträge, die sich wicderum zur Hauptsache an Schüler und Schülerinnen der Oberstufe von Mittelschulen und an die Lehrerschaft (Chemie, Biologie) richten. Eine Programmkommission aus Vertretern von Gymnasien, Hochschulen, Industrie und Presse hat sich für ein aktuelles Thema aus den Domänen Chemie - Biologie ... Physiologie - Verhaltensforschung entschieden. Es sollte nicht nur junge Menschen faszinieren und ihre Interessen wecken, sondern auch weiteren Teilnehmern z. B. Chemikern, Biologen - wertvolle Informationen vermittein.
Der Eintritt zu den Vorträgen, die von Dias, Filmen und Videos begleitet werden, ist für alle Interessenten frei. Eine dem Thema gewidmete Sondernummer der Zeitschrift für Naturwissenschaftslehrer 'Chemie und Biologie' wird vor dem Vortragsreferat aufliegen. Falls es die Zeit erlaubt, sollen auch Fragen an die Referenten gestellt werden können.

\section{Programm}

Chemische Signale und ihre Empfänger

Tagungsleiter: Prof. W. Simon, Laboratorium für Organische Chemie der ETH Zürich

Begrüssung und Einführung durch den Tagungsleiter

Dr. U. Burckhardt, CIBA-GEIGY AG, Basel Die Sprache der Kirschenfliege

Prof. H.J. Bestmann, Universität Erlangen, BRD Chemische Informationsübermittlung bei Insekten

Dozenten, Assistenten (Universitäten, ETH, HTL) die eine Gruppe (Doktoranden, Studenten) formieren können, erhalten Gratiskarten für den Besuch der Fachmesse ILMAC '90 (23.-26. Oktober 1990) an einem beliebigen Tag. Aus organisatorischen Gründen ist dafür allerdings eine schriftliche Anmeldung zur Teilnahme an die folgende Adresse bis zum 14. Oklober 1990 unter Angabe der Teilnehmerzahl und einer Empfangsadresse für den Versand erforderlich:

Für das CSC: Dr. A. Fürst, KfO

F. Hoffmann-La Roche AG, 71/113 Postfach

4002 Basel
ILMAC

11. Internationale

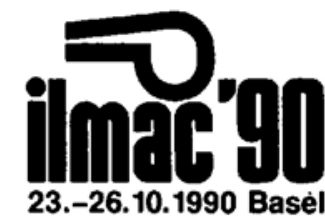

Chemie-Fachmesse

Ein Treffpunkt von Technik und Wissenschaft

Veranstaltet vom Schweizerischen Chemikerverband (SChV) findet die ILMAC - Internationale Chemie-Fachmesse - vom 23. bis 26 . Oktober' 1990 bereits zum 11. Mal in Basel im Rahmen der Schweizer Mustermesse statt. Thre Schwerpunkte sind Laborato riums- und Verfahrenstechnik, Messtechnik und Automatik. Die Fachmesse wird begleitet vom ILMACKongress. Beide Veranstaltungen stehen unter den Generalthema 'Neue Werkstoffe - neue Technologien'.

Uber 450 Aussteller werden an der ILMAC ihre Produkte und Dienstleistungen präsentieren. $\mathrm{Zu}$ den Ausstellungs-Schwerpunkten gehören erstmals auch Laboreinrichtungen für Arztpraxen. Weiter zählen dazu Biotechnologie, Isotopen und Radiochemie, Verfahrens- und Automationstechnik, Laborausstattung und -messtechnik sowie Sicherheit, Umweltschutz, Ausbildung und Institutionen. Die ILMAC gehört zu den international bedeutendsten europäischen Chemicfachmessen und findet alle drei Jahre statt. Sic schafft das Forum, den Markt, auf dem Innovationen dem Praktiker vorgestellt werden, auf dem der Forscher seine Bedürfnisse nach Ausrüstungen und Messgeräten anbringen und unter dem vielfältigen Angebot auswählen kann.

Eine Sonderschau steht unter dem Titel Explosionsschutz und wird gemeinsam vom Schweizerischen Ver- 
deutung zugesprochen wird und sie im geeigneten Umfeld ihre Stärke ausspielen und ihre wahre wirtschaftliche Bedeutung unter Beweis stellen kann.

Kein Land wie die Schweiz weist ein derart hohes analytisches Know-how pro Kapita auf, und doch stimmen die Voraussetzungen für eine wahrhaftig blühende Analytik nicht. An den Hochschulen fristet die analytische Forschung und Ausbildung mit wenigen Ausnahmen immer noch ein Schattendasein, und die Schweiz hat es auch nicht verstanden, ihr analytisches 'Know-how' in Gestalt der Instrumentalanalytik zu vermarkten, obwohl gerade mit dem Standort Schweiz die denkbar besten Voraussetzungen anzutreffen waren und immer noch anzutreffen sind, so zum Beispiel auf den Neuigkeitsgebieten der chemischen Sensoren und Biosensoren und der oben erwähnten analytischen Biotechnologie und analytischen Molekularbiologie, die für die immer wichtiger werdende medizinische Diagnostik und klinischen Untersuchungen von beachtlicher wirtschaftlicher Bedeutung sein könnte.

Im Lichte der bevorstehenden Fusion zwischen der Schweizerischen Chemischen Gesellschaft und dem Schweizerischen Chemiker-Verband und aufgrund einer durch solche Gedanken ausgelösten Analyse, die von einer aus einem halben Dutzend namhafter Schweizer Analytiker zusammengesetzten Gruppe am 15. November 1989 in Bern vorgenommen wurde, gründete diese Versammlung ein Interes- senkomitee, das Schweizerische Komitee für Analytische Chemie (Comité Suisse de Chimie Analytique, CSCA). In der Zwischenzeit fanden zwei weitere Sitzungen des CSCA statt, wobei der Teilnehmerkreis wesentlich erweitert wurde und den Worten auch Taten folgten.

Im CSCA sind die Hochschulen, eidgenössischen Anstalten und die chemische Industrie repräsentativ vertreten. Auch die einzelnen analytischen Interessengruppen und professionellen Vereinigungen sind durch ihre Präsidenten bestens vertreten. Es wurde versucht, andere Industriezweige, die klinische, medizinische Chemie und Lebensmittelchemie zu berücksichtigen, und es wurde Gewicht darauf gelegt, dass die französisch sprechende Schweiz wie die Deutschschweiz vertreten ist. Noch ist es bis jetzt nicht gelungen, im CSCA einen Vertreter aus der italienischen Schweiz engagieren zu können.

Das CSCA will sich für die Förderung der analytischen Chemie im weitesten Sinne, die Bildung und Weiterbildung auf dem Gebiet der analytischen Chemie einsetzen und in diesem Sinne aktiv werden. Es geht aber auch darum, im Rahmen der interdisziplinären Natur der modernen Analytik entsprechende Öffentlichkeitsarbeit zu leisten, gemeinsame Ziele der verschiedenen analytischen Disziplinen zu finden und diese gemeinsam anzupeilen.

Bereits anlässlich der Gründungssitzung beschloss das CSCA, der Schweizerischen Chemischen Gesellschaft das Gesuch zu stellen, an der Herbstversammlung vom 19. Oktober 1990 in Bern ein ganztägiges Analytikseminar durchführen zu dürfen. Diesem Antrag wurde entsprochen und durch ein Dutzend Vorträge und über dreissig Posters wird es gelingen, ein repräsentatives Schaufenster der schweizerischen Analytischen Chemie vorzuführen. Dabei kommen auf Einladung des CSCA auch zwei namhafte, international bekannte Analytiker zu Wort. Professor Csaba Horvath von der Yale University in New Haven, Connecticut, ein Pionier der LC, wird über die Möglichkeiten der ultraschnellen LC referieren, während Professor Shigeru Terabe vom Himeji Institute of Technology, Shosha, Himeji, Hyogo in Japan, bekannt für seine Arbeiten auf dem Gebiet der 'Micellar Electrokinetic Chromatography' (MECC), über neuste Entwicklungen auf diesem Spezialgebiet der Kapillarelektrophorese mit Micellen berichten wird.

Mit dem CSCA, einem reinen Interessenkomitee ohne Vereinsstatut, wird versucht, der modernen Analytik vermehrt Bedeutung zu verhelfen und der schweizerischen 'High Tech'-Analytik unter Umständen auch eine verbesserte wirtschaftliche Grundlage zu verschaffen. Das CSCA ist gewillt, die Zeitschrift CHIMIA vermehrt als Medium zur Exposition solcher Leistungen zu benutzen und diese als Sprachrohr zur Kommunikation von analytischen Bedürfnissen zu gebrauchen.

\section{Schweizerisches Komitee für Chemie Comité Suisse de la Chimie (CSC)}

Das Comité Suisse de la Chimie (CSC) ist die Dachorganisation von Chemischen Vereinigungen in der Schweiz. In dieser Funktion übernimmt es vor allem Aufgaben, die den Rahmen der einzelnen Mitgliedgesellschaften übersteigen oder von allgemeinem Interesse für die Chemie sind (gesamtschweizerische Koordinationsaufgaben, insbesondere auf den Gebieten Offentlichkeitsarbeit und Chemieunterricht; Landesvertretungen bei internationalen Organisationen).

ILMAC' '90:

Informationsveranstaltung

\section{Dienstag, 23. Oktober 1990, 15.00 Uhr Kongresszentrum der Schweizer Mustermesse Basel}

Nach den grossen Erfolgen in den Jahren 1984 und 1987 - jewcils an dic 1000 Teilnehmer, die meisten aus der Nordwestschweiz - organisiert das CSC auch heuer Vorträge, die sich wicderum zur Hauptsache an Schüler und Schülerinnen der Oberstufe von Mittelschulen und an die Lehrerschaft (Chemie, Biologie) richten. Eine Programmkommission aus Vertretern von Gymnasien, Hochschulen, Industrie und Presse hat sich für ein aktuelles Thema aus den Domänen Chemie - Biologie ... Physiologie - Verhaltensforschung entschieden. Es sollte nicht nur junge Menschen faszinieren und ihre Interessen wecken, sondern auch weiteren Teilnehmern z. B. Chemikern, Biologen - wertvolle Informationen vermittein.
Der Eintritt zu den Vorträgen, die von Dias, Filmen und Videos begleitet werden, ist für alle Interessenten frei. Eine dem Thema gewidmete Sondernummer der Zeitschrift für Naturwissenschaftslehrer 'Chemie und Biologie' wird vor dem Vortragsreferat aufliegen. Falls es die Zeit erlaubt, sollen auch Fragen an die Referenten gestellt werden können.

\section{Programm}

Chemische Signale und ihre Empfänger

Tagungsleiter: Prof. W. Simon, Laboratorium für Organische Chemie der ETH Zürich

Begrüssung und Einführung durch den Tagungsleiter

Dr. U. Burckhardt, CIBA-GEIGY AG, Basel Die Sprache der Kirschenfliege

Prof. H.J. Bestmann, Universität Erlangen, BRD Chemische Informationsübermittlung bei Insekten

Dozenten, Assistenten (Universitäten, ETH, HTL) die eine Gruppe (Doktoranden, Studenten) formieren können, erhalten Gratiskarten für den Besuch der Fachmesse ILMAC '90 (23.-26. Oktober 1990) an einem beliebigen Tag. Aus organisatorischen Gründen ist dafür allerdings eine schriftliche Anmeldung zur Teilnahme an die folgende Adresse bis zum 14. Oklober 1990 unter Angabe der Teilnehmerzahl und einer Empfangsadresse für den Versand erforderlich:

Für das CSC: Dr. A. Fürst, KfO

F. Hoffmann-La Roche AG, 71/113 Postfach

4002 Basel
ILMAC

11. Internationale

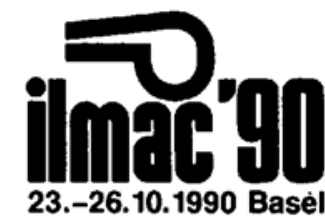

Chemie-Fachmesse

Ein Treffpunkt von Technik und Wissenschaft

Veranstaltet vom Schweizerischen Chemikerverband (SChV) findet die ILMAC - Internationale Chemie-Fachmesse - vom 23. bis 26 . Oktober' 1990 bereits zum 11. Mal in Basel im Rahmen der Schweizer Mustermesse statt. Thre Schwerpunkte sind Laborato riums- und Verfahrenstechnik, Messtechnik und Automatik. Die Fachmesse wird begleitet vom ILMACKongress. Beide Veranstaltungen stehen unter den Generalthema 'Neue Werkstoffe - neue Technologien'.

Uber 450 Aussteller werden an der ILMAC ihre Produkte und Dienstleistungen präsentieren. $\mathrm{Zu}$ den Ausstellungs-Schwerpunkten gehören erstmals auch Laboreinrichtungen für Arztpraxen. Weiter zählen dazu Biotechnologie, Isotopen und Radiochemie, Verfahrens- und Automationstechnik, Laborausstattung und -messtechnik sowie Sicherheit, Umweltschutz, Ausbildung und Institutionen. Die ILMAC gehört zu den international bedeutendsten europäischen Chemicfachmessen und findet alle drei Jahre statt. Sic schafft das Forum, den Markt, auf dem Innovationen dem Praktiker vorgestellt werden, auf dem der Forscher seine Bedürfnisse nach Ausrüstungen und Messgeräten anbringen und unter dem vielfältigen Angebot auswählen kann.

Eine Sonderschau steht unter dem Titel Explosionsschutz und wird gemeinsam vom Schweizerischen Ver- 
band für Umwelttechnik und dem Schweizerischen Elektrotechnischen Komitee (CES) Fachkommission 31 realisiert. Gezeigt werden neue Produkte und Technologien für den Einsatz elektrischer Betriebsmittel in explosionsgefährdeten Bereichen. Während der Dauer der Sonderschau stehen den Besuchern Mitglieder des Eidg. Starkstrominspektorates, des SEV (Schweizerischer Elektrotechnischer Verein), der SUVA und der Fachgruppe 31 für Fachgespräche und Auskünfte zur Verfügung.

Ein Hauptanliegen der Messe ist das Aufzeigen neuer Möglichkeiten und verbesserter Technologien zum Schutz von Mensch und Umwelt. In diese Richtung zielt nicht nur die Sonderschau. Das Thema der Messe verweist generell darauf, schaffen doch neue Werkstoffe wie auch neue Technologien die Grundlage für verfeinerte Methoden der Messung und der Kontrolle zum Schutz des Arbeitsplatzes und der Umwelt in der Produktion. Die ILMAC nimmt damit eine Herausforderung auf, welche mit der Diskussion um die Verantwortung der chemischen Industrie und der $\mathrm{Be}$ hörden verbunden ist.

Am begleitenden Kongress - dem 9. Basler Treffen für chemische Technik - finden zahlreiche Fachverbände den Rahmen zur Durchführung von Tagungen Wissenschaftler und Techniker aus aller Welt werden hier über den aktuellen Stand von Technik und Forschung berichten und diskutieren. Dem Besucher der ILMAC wird damit eine Bereicherung des Messebesuchs geboten.

\section{Vertreter der Schweiz wird Präsident des} europäischen Pharmaverbandes (EFPIA)

1.C. - Dr. Armin Kessler, Verwaltungsrat und Konzernleitungsmitglied der F. Hoffmann-La Roche AG, Basel, wurde anlässlich der EFPIA-Generalversammlung in Heidelberg einstimmig zum neuen Präsidenten für die Amtszeit 1990-92 gewählt. Kessler folgt auf $\mathrm{Dr}$ Alberto Aleotti (Menarini SpA) in einer Phase, die im Blick auf die Vollendung des EG-Binnenmarktes und die EWR-Verhandlungen für die pharmazeutische Industrie Europas von besonderer Bedeutung sein wird. Die EFPIA mit Sitz in Brüssel ist der europäische Pharmaverband, dem auch die Schweizerische Gesellschaft für Chemische Industrie (SGCI) als Vollmitglied angehört.

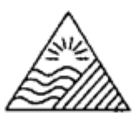

Internationa: Association of Environ mentel Analytical Chemistry Unter the patronage of the Swiss Feder

\section{2nd Soil Residue Analysis Workshop}

March 11-13, 1991

Ecole Polytechnique Fédérale de Lausanne (EPFL), Switzerland

Organization and information: Registration and Scientific Programme, Prof. Dr. Joseph Tarradellas, Chairman, IGE-EPFL, CH-1015 Lausanne, tel. (21) 693.27.12, fax. (21) 693.27.27
This Workshop is organized by the International Association of Environmental Analytical Chemistry and the Deparment of Analyticel Inorganic and Applied Chemistry of the University of Geneva. Swizertiand

Unter the Patronage of the Swiss Federal Otfice for the Environment, Forest and Landscape, Berne

\section{4th Hans Wolfgang Nürnberg \\ Memorial Workshop}

Toxic Metal Compounds

(Interrelation between Chemistry and Biology)

Congress-Center, CH-1865 Les Diablerets (Switzerland), March 4-8, 199

Organization and Information: Secretary and Address before and after the Workshop: Dr. Ernest Merian, Im Kirsgarten 22, CH-4106 Therwil (Switzerland), Tel. .. 4!-61-732950 (after 22nd April 1991 . 41-61-721 2950)

\section{Personalia}

\section{Geburtstage}

Alfred Hofstetter, Dr. sc. techn., Zürich, Mitglied des SChV, feiert am 3.10.90 seinen 60. Geburtstag.

Henri C. Silberman, Dr. chem., Laufen, Mitglied des SChV, feiert am 5.10 .90 seinen 65 . Geburtstag.

Rolf Schmid, Dr. Ing. Chem., Schwarzenburg, Mitglied des SChV, feiert am 6.10.90 seinen 65. Geburtstag.

Oswald Benz, Chemiker HTL, Grafenried, Mitglied des SChV, feiert am 11.10 .90 seinen 60 . Geburtstag.

Walter Frey, Dr. Ing. Chem., Muttenz, Mitglied des SChV, feiert am 12.10.90 seinen 75. Geburtstag.

Werner Manz, Dr. phil. II, Basel, Mitglied des SChV, feiert am 17.10.90 seinen 70. Geburtstag.

Ernst Kägi, Chemiker HTL, Monthey, Mitglied des SChV, feiert am 18.10 .90 seinen 60 . Geburtstag.

Max Sahli, Dr. phil., Chemiker, Kehrsatz, Mitglied des SChV, feiert am 23.10.90 seinen 65. Geburtstag.

Peter Jakober, Dr. sc. nat., Burgdorf, Mitglied des SChV, feiert am 24.10.90 seinen 60. Geburtstag.

Richard Neidlein, Prof. Dr., Heidelberg, Mitglied des SChV, feiert am 25.10 .90 seinen 60. Geburtstag.

U. Waltersperger, Chemiker HTL, Gossau, Mitglied des SChV, feiert am 29.10.90 seinen 65. Geburtstag.

\section{Neue Mitglieder}

Vogt-Schild AG, Druck und Verlag, Zuchwilerstrasse 21, 4501 Solothurn.

\section{Zusammenarbeit zwischen Roche und Western Capital für eine orale Form von Alpha-Interferon}

F. Hoffmann-La Roche AG, Basel, und die Western Capital Group (WCL) mit ihrer englischen PharmaTochtergesellschaft Cortecs Ltd. in London haben ein Abkommen über dic Zusammenarbeit zur Entwicklung und Prüfung einer oralen Form von alpha-Interferon unterzeichnet. Das Roche Präparat 'Roferon'-A wird hierbei zusammen mit dem Verabreichungssystem der WCL verwendet. Die Zusammenarbeit beruht auf früheren, von Cortecs durchgeführten klinischen Studien mit alpha-Interferon.

Interferone sind natürlich vorkommende Proteine oder Eiweisse. 'Roferon'-A ist das von Roche mittels Gentechnik produzierte Human-Interferon-alfa-2a; es ist in vielen Ländern als Mittel zur Behandlung von Haarzell-Leukämie, des durch AIDS verursachten Kaposi-Sarkoms und bösartiger Melanome zugelassen. Als weitere Indikationen werden derzeit registriert: chronische Hepatitis-B, kutanes T-Zell-Lymphom, chronisch-myeloische Leukämie und Nierenzellkrebs. Ausserdem wird 'Roferon'-A bei zusätzlichen Indikationen als Einzelsubstanz oder in Verbindung mit anderen Substanzen klinisch geprüft, so z. B. chronische Hepatitis-C, Darmkrebs sowie Lungenkrebs. Die Entwicklung einer oralen Form des Präparates könnte dic Behandlung dieser Krankheiten verbessern und den Alpha-Interferon-Markt erweitern.

In einer davon unabhängigen, zusätzlichen Entwicklung haben WCL und Cortecs im Mai 1990 bereits eine orale Form von Erythropoietin, dem kolonienstimulierenden Faktor roter Blutzellen, erfolgreich geprüft. Andere therapeutische Proteine, welche mit dem für grosse Moleküle bestimmten oralen Verabreichungssystem der WCL-Gruppe erfolgreich getestet wurden, schliessen Insulin, Calcitonin sowie menschliches Wachstumshormon ein.

\section{Fourth European Symposium on Radiopharmacy and Radiopharmaceuticals}

Baden (near Zürich), Switzerland Mai 1-4, 1991

Organized by the Radiopharmacy Group of the Swiss Society of Nuclear Medicine under the auspices of the EANM Taskgroup on Radiopharmaceuticals (Chairman: Prof. Dr. P. H. Cox)

The Fourth European Symposium will review recent developments in radiopharmacy and current research on radiopharmaceuticals. The scope will be expanded to include the pharmacokinetics and metabolism of radiopharmaceuticals, and the use of radioactive materials in drug formulation studies.

Secretary: c/o Radiopharmacy Division, Paul Scherrer Institutc, CH-5232 Villigen PSI, Switzerland, Telephone 0569928 14, Telex 827418 (psir ch), Telefax 056 982635 .

\section{EURO FOOD TOX III}

Schweizerische Chemische Gesellschaft Schweizerischer Chemiker-Verband

\section{Herbstversammlung in Bern}

Freitag, 19. Oktober 1990

Chemische Institute der Universität Bern, Freiestrasse 3

Programm $08.30-09.00$

09.00-09.30 Plenarvortrag des Werner-Preisträgers 1990 Dr. H. Frei, University of California Berkeley/USA

'Chemistry with red and near infrared light'

09.40-18.00 Kurzvorträge der Sektion für Organische Chemie

09.40-17.45 Vorträge der Sektion für Medizinische Chemie
09.40-15.40 Kurzvorträge der Sektion für Physikalische Chemie

14.20-17.00 Kurzvorträge der Sektion für Computerunterstützte Chemie

09.00-12.30 Minisymposium 'Recent Developments in Organometallic Chemistry and Homogeneous Catalysis'

14.30-16.30 Postersession der Sektion für Anorganische Chemie und Koordinationschemic

10.30-17.40 Seminar 'Analytische Chemie' 13.30-15.00 Postersession 'Analytische Chemie'

Information/Kontakt:

Dr. E. Zass, Sekretär SCG

ETH Zürich, Universitätstr. 16, CH-8092 Zürich
Interdisciplinary Conference on the Effects of Food on the Immune and Hormonal Systems sponsored by The European Society of Toxicology, The Federation of European Chemical Societies: Working Party on Food Chemistry, The Federation of European Nutrition Societies.

May 23-24, 1991, Zurich/Switzerland

Information: EURO FOOD TOX III, Sekretarial, MGB Zentrallabor, Postfach 266, 8031 Zürich, Switzerland.

\section{Der Schweizerische Schulrat}

hat den Ruzicka-Preis 1990 an Dr. Charles Fehr, Firmenich SA, Research Laboratories, Genf, verliehen. Anlässlich der Preisverleihung vom 10. September 1990 an der ETH Zürich hat der Preisträger folgenden Vortrag gehalten: Enantioselektive Protonierung von Enolaten zur Synthese von Naturstoffen. 
band für Umwelttechnik und dem Schweizerischen Elektrotechnischen Komitee (CES) Fachkommission 31 realisiert. Gezeigt werden neue Produkte und Technologien für den Einsatz elektrischer Betriebsmittel in explosionsgefährdeten Bereichen. Während der Dauer der Sonderschau stehen den Besuchern Mitglieder des Eidg. Starkstrominspektorates, des SEV (Schweizerischer Elektrotechnischer Verein), der SUVA und der Fachgruppe 31 für Fachgespräche und Auskünfte zur Verfügung.

Ein Hauptanliegen der Messe ist das Aufzeigen neuer Möglichkeiten und verbesserter Technologien zum Schutz von Mensch und Umwelt. In diese Richtung zielt nicht nur die Sonderschau. Das Thema der Messe verweist generell darauf, schaffen doch neue Werkstoffe wie auch neue Technologien die Grundlage für verfeinerte Methoden der Messung und der Kontrolle zum Schutz des Arbeitsplatzes und der Umwelt in der Produktion. Die ILMAC nimmt damit eine Herausforderung auf, welche mit der Diskussion um die Verantwortung der chemischen Industrie und der $\mathrm{Be}$ hörden verbunden ist.

Am begleitenden Kongress - dem 9. Basler Treffen für chemische Technik - finden zahlreiche Fachverbände den Rahmen zur Durchführung von Tagungen Wissenschaftler und Techniker aus aller Welt werden hier über den aktuellen Stand von Technik und Forschung berichten und diskutieren. Dem Besucher der ILMAC wird damit eine Bereicherung des Messebesuchs geboten.

\section{Vertreter der Schweiz wird Präsident des} europäischen Pharmaverbandes (EFPIA)

1.C. - Dr. Armin Kessler, Verwaltungsrat und Konzernleitungsmitglied der F. Hoffmann-La Roche AG, Basel, wurde anlässlich der EFPIA-Generalversammlung in Heidelberg einstimmig zum neuen Präsidenten für die Amtszeit 1990-92 gewählt. Kessler folgt auf $\mathrm{Dr}$ Alberto Aleotti (Menarini SpA) in einer Phase, die im Blick auf die Vollendung des EG-Binnenmarktes und die EWR-Verhandlungen für die pharmazeutische Industrie Europas von besonderer Bedeutung sein wird. Die EFPIA mit Sitz in Brüssel ist der europäische Pharmaverband, dem auch die Schweizerische Gesellschaft für Chemische Industrie (SGCI) als Vollmitglied angehört.

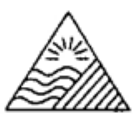

Internationa: Association of Environ mentel Analytical Chemistry Unter the patronage of the Swiss Feder

\section{2nd Soil Residue Analysis Workshop}

March 11-13, 1991

Ecole Polytechnique Fédérale de Lausanne (EPFL), Switzerland

Organization and information: Registration and Scientific Programme, Prof. Dr. Joseph Tarradellas, Chairman, IGE-EPFL, CH-1015 Lausanne, tel. (21) 693.27.12, fax. (21) 693.27.27
This Workshop is organized by the International Association of Environmental Analytical Chemistry and the Deparment of Analyticel Inorganic and Applied Chemistry of the University of Geneva. Swizertiand

Unter the Patronage of the Swiss Federal Otfice for the Environment, Forest and Landscape, Berne

\section{4th Hans Wolfgang Nürnberg \\ Memorial Workshop}

Toxic Metal Compounds

(Interrelation between Chemistry and Biology)

Congress-Center, CH-1865 Les Diablerets (Switzerland), March 4-8, 199

Organization and Information: Secretary and Address before and after the Workshop: Dr. Ernest Merian, Im Kirsgarten 22, CH-4106 Therwil (Switzerland), Tel. .. 4!-61-732950 (after 22nd April 1991 . 41-61-721 2950)

\section{Personalia}

\section{Geburtstage}

Alfred Hofstetter, Dr. sc. techn., Zürich, Mitglied des SChV, feiert am 3.10.90 seinen 60. Geburtstag.

Henri C. Silberman, Dr. chem., Laufen, Mitglied des SChV, feiert am 5.10 .90 seinen 65 . Geburtstag.

Rolf Schmid, Dr. Ing. Chem., Schwarzenburg, Mitglied des SChV, feiert am 6.10.90 seinen 65. Geburtstag.

Oswald Benz, Chemiker HTL, Grafenried, Mitglied des SChV, feiert am 11.10 .90 seinen 60 . Geburtstag.

Walter Frey, Dr. Ing. Chem., Muttenz, Mitglied des SChV, feiert am 12.10.90 seinen 75. Geburtstag.

Werner Manz, Dr. phil. II, Basel, Mitglied des SChV, feiert am 17.10.90 seinen 70. Geburtstag.

Ernst Kägi, Chemiker HTL, Monthey, Mitglied des SChV, feiert am 18.10 .90 seinen 60 . Geburtstag.

Max Sahli, Dr. phil., Chemiker, Kehrsatz, Mitglied des SChV, feiert am 23.10.90 seinen 65. Geburtstag.

Peter Jakober, Dr. sc. nat., Burgdorf, Mitglied des SChV, feiert am 24.10.90 seinen 60. Geburtstag.

Richard Neidlein, Prof. Dr., Heidelberg, Mitglied des SChV, feiert am 25.10 .90 seinen 60. Geburtstag.

U. Waltersperger, Chemiker HTL, Gossau, Mitglied des SChV, feiert am 29.10.90 seinen 65. Geburtstag.

\section{Neue Mitglieder}

Vogt-Schild AG, Druck und Verlag, Zuchwilerstrasse 21, 4501 Solothurn.

\section{Zusammenarbeit zwischen Roche und Western Capital für eine orale Form von Alpha-Interferon}

F. Hoffmann-La Roche AG, Basel, und die Western Capital Group (WCL) mit ihrer englischen PharmaTochtergesellschaft Cortecs Ltd. in London haben ein Abkommen über dic Zusammenarbeit zur Entwicklung und Prüfung einer oralen Form von alpha-Interferon unterzeichnet. Das Roche Präparat 'Roferon'-A wird hierbei zusammen mit dem Verabreichungssystem der WCL verwendet. Die Zusammenarbeit beruht auf früheren, von Cortecs durchgeführten klinischen Studien mit alpha-Interferon.

Interferone sind natürlich vorkommende Proteine oder Eiweisse. 'Roferon'-A ist das von Roche mittels Gentechnik produzierte Human-Interferon-alfa-2a; es ist in vielen Ländern als Mittel zur Behandlung von Haarzell-Leukämie, des durch AIDS verursachten Kaposi-Sarkoms und bösartiger Melanome zugelassen. Als weitere Indikationen werden derzeit registriert: chronische Hepatitis-B, kutanes T-Zell-Lymphom, chronisch-myeloische Leukämie und Nierenzellkrebs. Ausserdem wird 'Roferon'-A bei zusätzlichen Indikationen als Einzelsubstanz oder in Verbindung mit anderen Substanzen klinisch geprüft, so z. B. chronische Hepatitis-C, Darmkrebs sowie Lungenkrebs. Die Entwicklung einer oralen Form des Präparates könnte dic Behandlung dieser Krankheiten verbessern und den Alpha-Interferon-Markt erweitern.

In einer davon unabhängigen, zusätzlichen Entwicklung haben WCL und Cortecs im Mai 1990 bereits eine orale Form von Erythropoietin, dem kolonienstimulierenden Faktor roter Blutzellen, erfolgreich geprüft. Andere therapeutische Proteine, welche mit dem für grosse Moleküle bestimmten oralen Verabreichungssystem der WCL-Gruppe erfolgreich getestet wurden, schliessen Insulin, Calcitonin sowie menschliches Wachstumshormon ein.

\section{Fourth European Symposium on Radiopharmacy and Radiopharmaceuticals}

Baden (near Zürich), Switzerland Mai 1-4, 1991

Organized by the Radiopharmacy Group of the Swiss Society of Nuclear Medicine under the auspices of the EANM Taskgroup on Radiopharmaceuticals (Chairman: Prof. Dr. P. H. Cox)

The Fourth European Symposium will review recent developments in radiopharmacy and current research on radiopharmaceuticals. The scope will be expanded to include the pharmacokinetics and metabolism of radiopharmaceuticals, and the use of radioactive materials in drug formulation studies.

Secretary: c/o Radiopharmacy Division, Paul Scherrer Institutc, CH-5232 Villigen PSI, Switzerland, Telephone 0569928 14, Telex 827418 (psir ch), Telefax 056 982635 .

\section{EURO FOOD TOX III}

Schweizerische Chemische Gesellschaft Schweizerischer Chemiker-Verband

\section{Herbstversammlung in Bern}

Freitag, 19. Oktober 1990

Chemische Institute der Universität Bern, Freiestrasse 3

Programm $08.30-09.00$

09.00-09.30 Plenarvortrag des Werner-Preisträgers 1990 Dr. H. Frei, University of California Berkeley/USA

'Chemistry with red and near infrared light'

09.40-18.00 Kurzvorträge der Sektion für Organische Chemie

09.40-17.45 Vorträge der Sektion für Medizinische Chemie
09.40-15.40 Kurzvorträge der Sektion für Physikalische Chemie

14.20-17.00 Kurzvorträge der Sektion für Computerunterstützte Chemie

09.00-12.30 Minisymposium 'Recent Developments in Organometallic Chemistry and Homogeneous Catalysis'

14.30-16.30 Postersession der Sektion für Anorganische Chemie und Koordinationschemic

10.30-17.40 Seminar 'Analytische Chemie' 13.30-15.00 Postersession 'Analytische Chemie'

Information/Kontakt:

Dr. E. Zass, Sekretär SCG

ETH Zürich, Universitätstr. 16, CH-8092 Zürich
Interdisciplinary Conference on the Effects of Food on the Immune and Hormonal Systems sponsored by The European Society of Toxicology, The Federation of European Chemical Societies: Working Party on Food Chemistry, The Federation of European Nutrition Societies.

May 23-24, 1991, Zurich/Switzerland

Information: EURO FOOD TOX III, Sekretarial, MGB Zentrallabor, Postfach 266, 8031 Zürich, Switzerland.

\section{Der Schweizerische Schulrat}

hat den Ruzicka-Preis 1990 an Dr. Charles Fehr, Firmenich SA, Research Laboratories, Genf, verliehen. Anlässlich der Preisverleihung vom 10. September 1990 an der ETH Zürich hat der Preisträger folgenden Vortrag gehalten: Enantioselektive Protonierung von Enolaten zur Synthese von Naturstoffen. 
band für Umwelttechnik und dem Schweizerischen Elektrotechnischen Komitee (CES) Fachkommission 31 realisiert. Gezeigt werden neue Produkte und Technologien für den Einsatz elektrischer Betriebsmittel in explosionsgefährdeten Bereichen. Während der Dauer der Sonderschau stehen den Besuchern Mitglieder des Eidg. Starkstrominspektorates, des SEV (Schweizerischer Elektrotechnischer Verein), der SUVA und der Fachgruppe 31 für Fachgespräche und Auskünfte zur Verfügung.

Ein Hauptanliegen der Messe ist das Aufzeigen neuer Möglichkeiten und verbesserter Technologien zum Schutz von Mensch und Umwelt. In diese Richtung zielt nicht nur die Sonderschau. Das Thema der Messe verweist generell darauf, schaffen doch neue Werkstoffe wie auch neue Technologien die Grundlage für verfeinerte Methoden der Messung und der Kontrolle zum Schutz des Arbeitsplatzes und der Umwelt in der Produktion. Die ILMAC nimmt damit eine Herausforderung auf, welche mit der Diskussion um die Verantwortung der chemischen Industrie und der $\mathrm{Be}$ hörden verbunden ist.

Am begleitenden Kongress - dem 9. Basler Treffen für chemische Technik - finden zahlreiche Fachverbände den Rahmen zur Durchführung von Tagungen Wissenschaftler und Techniker aus aller Welt werden hier über den aktuellen Stand von Technik und Forschung berichten und diskutieren. Dem Besucher der ILMAC wird damit eine Bereicherung des Messebesuchs geboten.

\section{Vertreter der Schweiz wird Präsident des} europäischen Pharmaverbandes (EFPIA)

1.C. - Dr. Armin Kessler, Verwaltungsrat und Konzernleitungsmitglied der F. Hoffmann-La Roche AG, Basel, wurde anlässlich der EFPIA-Generalversammlung in Heidelberg einstimmig zum neuen Präsidenten für die Amtszeit 1990-92 gewählt. Kessler folgt auf $\mathrm{Dr}$ Alberto Aleotti (Menarini SpA) in einer Phase, die im Blick auf die Vollendung des EG-Binnenmarktes und die EWR-Verhandlungen für die pharmazeutische Industrie Europas von besonderer Bedeutung sein wird. Die EFPIA mit Sitz in Brüssel ist der europäische Pharmaverband, dem auch die Schweizerische Gesellschaft für Chemische Industrie (SGCI) als Vollmitglied angehört.

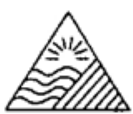

Internationa: Association of Environ mentel Analytical Chemistry Unter the patronage of the Swiss Feder

\section{2nd Soil Residue Analysis Workshop}

March 11-13, 1991

Ecole Polytechnique Fédérale de Lausanne (EPFL), Switzerland

Organization and information: Registration and Scientific Programme, Prof. Dr. Joseph Tarradellas, Chairman, IGE-EPFL, CH-1015 Lausanne, tel. (21) 693.27.12, fax. (21) 693.27.27
This Workshop is organized by the International Association of Environmental Analytical Chemistry and the Deparment of Analyticel Inorganic and Applied Chemistry of the University of Geneva. Swizertiand

Unter the Patronage of the Swiss Federal Otfice for the Environment, Forest and Landscape, Berne

\section{4th Hans Wolfgang Nürnberg \\ Memorial Workshop}

Toxic Metal Compounds

(Interrelation between Chemistry and Biology)

Congress-Center, CH-1865 Les Diablerets (Switzerland), March 4-8, 199

Organization and Information: Secretary and Address before and after the Workshop: Dr. Ernest Merian, Im Kirsgarten 22, CH-4106 Therwil (Switzerland), Tel. .. 4!-61-732950 (after 22nd April 1991 . 41-61-721 2950)

\section{Personalia}

\section{Geburtstage}

Alfred Hofstetter, Dr. sc. techn., Zürich, Mitglied des SChV, feiert am 3.10.90 seinen 60. Geburtstag.

Henri C. Silberman, Dr. chem., Laufen, Mitglied des SChV, feiert am 5.10 .90 seinen 65 . Geburtstag.

Rolf Schmid, Dr. Ing. Chem., Schwarzenburg, Mitglied des SChV, feiert am 6.10.90 seinen 65. Geburtstag.

Oswald Benz, Chemiker HTL, Grafenried, Mitglied des SChV, feiert am 11.10 .90 seinen 60 . Geburtstag.

Walter Frey, Dr. Ing. Chem., Muttenz, Mitglied des SChV, feiert am 12.10.90 seinen 75. Geburtstag.

Werner Manz, Dr. phil. II, Basel, Mitglied des SChV, feiert am 17.10.90 seinen 70. Geburtstag.

Ernst Kägi, Chemiker HTL, Monthey, Mitglied des SChV, feiert am 18.10 .90 seinen 60 . Geburtstag.

Max Sahli, Dr. phil., Chemiker, Kehrsatz, Mitglied des SChV, feiert am 23.10.90 seinen 65. Geburtstag.

Peter Jakober, Dr. sc. nat., Burgdorf, Mitglied des SChV, feiert am 24.10.90 seinen 60. Geburtstag.

Richard Neidlein, Prof. Dr., Heidelberg, Mitglied des SChV, feiert am 25.10 .90 seinen 60. Geburtstag.

U. Waltersperger, Chemiker HTL, Gossau, Mitglied des SChV, feiert am 29.10.90 seinen 65. Geburtstag.

\section{Neue Mitglieder}

Vogt-Schild AG, Druck und Verlag, Zuchwilerstrasse 21, 4501 Solothurn.

\section{Zusammenarbeit zwischen Roche und Western Capital für eine orale Form von Alpha-Interferon}

F. Hoffmann-La Roche AG, Basel, und die Western Capital Group (WCL) mit ihrer englischen PharmaTochtergesellschaft Cortecs Ltd. in London haben ein Abkommen über dic Zusammenarbeit zur Entwicklung und Prüfung einer oralen Form von alpha-Interferon unterzeichnet. Das Roche Präparat 'Roferon'-A wird hierbei zusammen mit dem Verabreichungssystem der WCL verwendet. Die Zusammenarbeit beruht auf früheren, von Cortecs durchgeführten klinischen Studien mit alpha-Interferon.

Interferone sind natürlich vorkommende Proteine oder Eiweisse. 'Roferon'-A ist das von Roche mittels Gentechnik produzierte Human-Interferon-alfa-2a; es ist in vielen Ländern als Mittel zur Behandlung von Haarzell-Leukämie, des durch AIDS verursachten Kaposi-Sarkoms und bösartiger Melanome zugelassen. Als weitere Indikationen werden derzeit registriert: chronische Hepatitis-B, kutanes T-Zell-Lymphom, chronisch-myeloische Leukämie und Nierenzellkrebs. Ausserdem wird 'Roferon'-A bei zusätzlichen Indikationen als Einzelsubstanz oder in Verbindung mit anderen Substanzen klinisch geprüft, so z. B. chronische Hepatitis-C, Darmkrebs sowie Lungenkrebs. Die Entwicklung einer oralen Form des Präparates könnte dic Behandlung dieser Krankheiten verbessern und den Alpha-Interferon-Markt erweitern.

In einer davon unabhängigen, zusätzlichen Entwicklung haben WCL und Cortecs im Mai 1990 bereits eine orale Form von Erythropoietin, dem kolonienstimulierenden Faktor roter Blutzellen, erfolgreich geprüft. Andere therapeutische Proteine, welche mit dem für grosse Moleküle bestimmten oralen Verabreichungssystem der WCL-Gruppe erfolgreich getestet wurden, schliessen Insulin, Calcitonin sowie menschliches Wachstumshormon ein.

\section{Fourth European Symposium on Radiopharmacy and Radiopharmaceuticals}

Baden (near Zürich), Switzerland Mai 1-4, 1991

Organized by the Radiopharmacy Group of the Swiss Society of Nuclear Medicine under the auspices of the EANM Taskgroup on Radiopharmaceuticals (Chairman: Prof. Dr. P. H. Cox)

The Fourth European Symposium will review recent developments in radiopharmacy and current research on radiopharmaceuticals. The scope will be expanded to include the pharmacokinetics and metabolism of radiopharmaceuticals, and the use of radioactive materials in drug formulation studies.

Secretary: c/o Radiopharmacy Division, Paul Scherrer Institutc, CH-5232 Villigen PSI, Switzerland, Telephone 0569928 14, Telex 827418 (psir ch), Telefax 056 982635 .

\section{EURO FOOD TOX III}

Schweizerische Chemische Gesellschaft Schweizerischer Chemiker-Verband

\section{Herbstversammlung in Bern}

Freitag, 19. Oktober 1990

Chemische Institute der Universität Bern, Freiestrasse 3

Programm $08.30-09.00$

09.00-09.30 Plenarvortrag des Werner-Preisträgers 1990 Dr. H. Frei, University of California Berkeley/USA

'Chemistry with red and near infrared light'

09.40-18.00 Kurzvorträge der Sektion für Organische Chemie

09.40-17.45 Vorträge der Sektion für Medizinische Chemie
09.40-15.40 Kurzvorträge der Sektion für Physikalische Chemie

14.20-17.00 Kurzvorträge der Sektion für Computerunterstützte Chemie

09.00-12.30 Minisymposium 'Recent Developments in Organometallic Chemistry and Homogeneous Catalysis'

14.30-16.30 Postersession der Sektion für Anorganische Chemie und Koordinationschemic

10.30-17.40 Seminar 'Analytische Chemie' 13.30-15.00 Postersession 'Analytische Chemie'

Information/Kontakt:

Dr. E. Zass, Sekretär SCG

ETH Zürich, Universitätstr. 16, CH-8092 Zürich
Interdisciplinary Conference on the Effects of Food on the Immune and Hormonal Systems sponsored by The European Society of Toxicology, The Federation of European Chemical Societies: Working Party on Food Chemistry, The Federation of European Nutrition Societies.

May 23-24, 1991, Zurich/Switzerland

Information: EURO FOOD TOX III, Sekretarial, MGB Zentrallabor, Postfach 266, 8031 Zürich, Switzerland.

\section{Der Schweizerische Schulrat}

hat den Ruzicka-Preis 1990 an Dr. Charles Fehr, Firmenich SA, Research Laboratories, Genf, verliehen. Anlässlich der Preisverleihung vom 10. September 1990 an der ETH Zürich hat der Preisträger folgenden Vortrag gehalten: Enantioselektive Protonierung von Enolaten zur Synthese von Naturstoffen. 
band für Umwelttechnik und dem Schweizerischen Elektrotechnischen Komitee (CES) Fachkommission 31 realisiert. Gezeigt werden neue Produkte und Technologien für den Einsatz elektrischer Betriebsmittel in explosionsgefährdeten Bereichen. Während der Dauer der Sonderschau stehen den Besuchern Mitglieder des Eidg. Starkstrominspektorates, des SEV (Schweizerischer Elektrotechnischer Verein), der SUVA und der Fachgruppe 31 für Fachgespräche und Auskünfte zur Verfügung.

Ein Hauptanliegen der Messe ist das Aufzeigen neuer Möglichkeiten und verbesserter Technologien zum Schutz von Mensch und Umwelt. In diese Richtung zielt nicht nur die Sonderschau. Das Thema der Messe verweist generell darauf, schaffen doch neue Werkstoffe wie auch neue Technologien die Grundlage für verfeinerte Methoden der Messung und der Kontrolle zum Schutz des Arbeitsplatzes und der Umwelt in der Produktion. Die ILMAC nimmt damit eine Herausforderung auf, welche mit der Diskussion um die Verantwortung der chemischen Industrie und der $\mathrm{Be}$ hörden verbunden ist.

Am begleitenden Kongress - dem 9. Basler Treffen für chemische Technik - finden zahlreiche Fachverbände den Rahmen zur Durchführung von Tagungen Wissenschaftler und Techniker aus aller Welt werden hier über den aktuellen Stand von Technik und Forschung berichten und diskutieren. Dem Besucher der ILMAC wird damit eine Bereicherung des Messebesuchs geboten.

\section{Vertreter der Schweiz wird Präsident des} europäischen Pharmaverbandes (EFPIA)

1.C. - Dr. Armin Kessler, Verwaltungsrat und Konzernleitungsmitglied der F. Hoffmann-La Roche AG, Basel, wurde anlässlich der EFPIA-Generalversammlung in Heidelberg einstimmig zum neuen Präsidenten für die Amtszeit 1990-92 gewählt. Kessler folgt auf $\mathrm{Dr}$ Alberto Aleotti (Menarini SpA) in einer Phase, die im Blick auf die Vollendung des EG-Binnenmarktes und die EWR-Verhandlungen für die pharmazeutische Industrie Europas von besonderer Bedeutung sein wird. Die EFPIA mit Sitz in Brüssel ist der europäische Pharmaverband, dem auch die Schweizerische Gesellschaft für Chemische Industrie (SGCI) als Vollmitglied angehört.

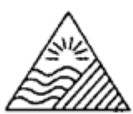

Internationa: Association of Environ mentel Analytical Chemistry Unter the patronage of the Swiss Feder

\section{2nd Soil Residue Analysis Workshop}

March 11-13, 1991

Ecole Polytechnique Fédérale de Lausanne (EPFL), Switzerland

Organization and information: Registration and Scientific Programme, Prof. Dr. Joseph Tarradellas, Chairman, IGE-EPFL, CH-1015 Lausanne, tel. (21) 693.27.12, fax. (21) 693.27.27
This Workshop is organized by the International Association of Environmental Analytical Chemistry and the Deparment of Analyticel Inorganic and Applied Chemistry of the University of Geneva. Swizertiand

Unter the Patronage of the Swiss Federal Otfice for the Environment, Forest and Landscape, Berne

\section{4th Hans Wolfgang Nürnberg \\ Memorial Workshop}

Toxic Metal Compounds

(Interrelation between Chemistry and Biology)

Congress-Center, CH-1865 Les Diablerets (Switzerland), March 4-8, 199

Organization and Information: Secretary and Address before and after the Workshop: Dr. Ernest Merian, Im Kirsgarten 22, CH-4106 Therwil (Switzerland), Tel. .. 4!-61-732950 (after 22nd April 1991 . 41-61-721 2950)

\section{Personalia}

\section{Geburtstage}

Alfred Hofstetter, Dr. sc. techn., Zürich, Mitglied des SChV, feiert am 3.10.90 seinen 60. Geburtstag.

Henri C. Silberman, Dr. chem., Laufen, Mitglied des SChV, feiert am 5.10 .90 seinen 65 . Geburtstag.

Rolf Schmid, Dr. Ing. Chem., Schwarzenburg, Mitglied des SChV, feiert am 6.10.90 seinen 65. Geburtstag.

Oswald Benz, Chemiker HTL, Grafenried, Mitglied des SChV, feiert am 11.10 .90 seinen 60 . Geburtstag.

Walter Frey, Dr. Ing. Chem., Muttenz, Mitglied des SChV, feiert am 12.10.90 seinen 75. Geburtstag.

Werner Manz, Dr. phil. II, Basel, Mitglied des SChV, feiert am 17.10.90 seinen 70. Geburtstag.

Ernst Kägi, Chemiker HTL, Monthey, Mitglied des SChV, feiert am 18.10 .90 seinen 60 . Geburtstag.

Max Sahli, Dr. phil., Chemiker, Kehrsatz, Mitglied des SChV, feiert am 23.10.90 seinen 65. Geburtstag.

Peter Jakober, Dr. sc. nat., Burgdorf, Mitglied des SChV, feiert am 24.10.90 seinen 60. Geburtstag.

Richard Neidlein, Prof. Dr., Heidelberg, Mitglied des SChV, feiert am 25.10 .90 seinen 60. Geburtstag.

U. Waltersperger, Chemiker HTL, Gossau, Mitglied des SChV, feiert am 29.10.90 seinen 65. Geburtstag.

\section{Neue Mitglieder}

Vogt-Schild AG, Druck und Verlag, Zuchwilerstrasse 21, 4501 Solothurn.

\section{Zusammenarbeit zwischen Roche und Western Capital für eine orale Form von Alpha-Interferon}

F. Hoffmann-La Roche AG, Basel, und die Western Capital Group (WCL) mit ihrer englischen PharmaTochtergesellschaft Cortecs Ltd. in London haben ein Abkommen über dic Zusammenarbeit zur Entwicklung und Prüfung einer oralen Form von alpha-Interferon unterzeichnet. Das Roche Präparat 'Roferon'-A wird hierbei zusammen mit dem Verabreichungssystem der WCL verwendet. Die Zusammenarbeit beruht auf früheren, von Cortecs durchgeführten klinischen Studien mit alpha-Interferon.

Interferone sind natürlich vorkommende Proteine oder Eiweisse. 'Roferon'-A ist das von Roche mittels Gentechnik produzierte Human-Interferon-alfa-2a; es ist in vielen Ländern als Mittel zur Behandlung von Haarzell-Leukämie, des durch AIDS verursachten Kaposi-Sarkoms und bösartiger Melanome zugelassen. Als weitere Indikationen werden derzeit registriert: chronische Hepatitis-B, kutanes T-Zell-Lymphom, chronisch-myeloische Leukämie und Nierenzellkrebs. Ausserdem wird 'Roferon'-A bei zusätzlichen Indikationen als Einzelsubstanz oder in Verbindung mit anderen Substanzen klinisch geprüft, so z. B. chronische Hepatitis-C, Darmkrebs sowie Lungenkrebs. Die Entwicklung einer oralen Form des Präparates könnte dic Behandlung dieser Krankheiten verbessern und den Alpha-Interferon-Markt erweitern.

In einer davon unabhängigen, zusätzlichen Entwicklung haben WCL und Cortecs im Mai 1990 bereits eine orale Form von Erythropoietin, dem kolonienstimulierenden Faktor roter Blutzellen, erfolgreich geprüft. Andere therapeutische Proteine, welche mit dem für grosse Moleküle bestimmten oralen Verabreichungssystem der WCL-Gruppe erfolgreich getestet wurden, schliessen Insulin, Calcitonin sowie menschliches Wachstumshormon ein.

\section{Fourth European Symposium on Radiopharmacy and Radiopharmaceuticals}

Baden (near Zürich), Switzerland Mai 1-4, 1991

Organized by the Radiopharmacy Group of the Swiss Society of Nuclear Medicine under the auspices of the EANM Taskgroup on Radiopharmaceuticals (Chairman: Prof. Dr. P. H. Cox)

The Fourth European Symposium will review recent developments in radiopharmacy and current research on radiopharmaceuticals. The scope will be expanded to include the pharmacokinetics and metabolism of radiopharmaceuticals, and the use of radioactive materials in drug formulation studies.

Secretary: c/o Radiopharmacy Division, Paul Scherrer Institutc, CH-5232 Villigen PSI, Switzerland, Telephone 0569928 14, Telex 827418 (psir ch), Telefax 056 982635 .

\section{EURO FOOD TOX III}

Schweizerische Chemische Gesellschaft Schweizerischer Chemiker-Verband

\section{Herbstversammlung in Bern}

Freitag, 19. Oktober 1990

Chemische Institute der Universität Bern, Freiestrasse 3

Programm $08.30-09.00$

09.00-09.30 Plenarvortrag des Werner-Preisträgers 1990 Dr. H. Frei, University of California Berkeley/USA

'Chemistry with red and near infrared light'

09.40-18.00 Kurzvorträge der Sektion für Organische Chemie

09.40-17.45 Vorträge der Sektion für Medizinische Chemie
09.40-15.40 Kurzvorträge der Sektion für Physikalische Chemie

14.20-17.00 Kurzvorträge der Sektion für Computerunterstützte Chemie

09.00-12.30 Minisymposium 'Recent Developments in Organometallic Chemistry and Homogeneous Catalysis'

14.30-16.30 Postersession der Sektion für Anorganische Chemie und Koordinationschemic

10.30-17.40 Seminar 'Analytische Chemie' 13.30-15.00 Postersession 'Analytische Chemie'

Information/Kontakt:

Dr. E. Zass, Sekretär SCG

ETH Zürich, Universitätstr. 16, CH-8092 Zürich
Interdisciplinary Conference on the Effects of Food on the Immune and Hormonal Systems sponsored by The European Society of Toxicology, The Federation of European Chemical Societies: Working Party on Food Chemistry, The Federation of European Nutrition Societies.

May 23-24, 1991, Zurich/Switzerland

Information: EURO FOOD TOX III, Sekretarial, MGB Zentrallabor, Postfach 266, 8031 Zürich, Switzerland.

\section{Der Schweizerische Schulrat}

hat den Ruzicka-Preis 1990 an Dr. Charles Fehr, Firmenich SA, Research Laboratories, Genf, verliehen. Anlässlich der Preisverleihung vom 10. September 1990 an der ETH Zürich hat der Preisträger folgenden Vortrag gehalten: Enantioselektive Protonierung von Enolaten zur Synthese von Naturstoffen. 
band für Umwelttechnik und dem Schweizerischen Elektrotechnischen Komitee (CES) Fachkommission 31 realisiert. Gezeigt werden neue Produkte und Technologien für den Einsatz elektrischer Betriebsmittel in explosionsgefährdeten Bereichen. Während der Dauer der Sonderschau stehen den Besuchern Mitglieder des Eidg. Starkstrominspektorates, des SEV (Schweizerischer Elektrotechnischer Verein), der SUVA und der Fachgruppe 31 für Fachgespräche und Auskünfte zur Verfügung.

Ein Hauptanliegen der Messe ist das Aufzeigen neuer Möglichkeiten und verbesserter Technologien zum Schutz von Mensch und Umwelt. In diese Richtung zielt nicht nur die Sonderschau. Das Thema der Messe verweist generell darauf, schaffen doch neue Werkstoffe wie auch neue Technologien die Grundlage für verfeinerte Methoden der Messung und der Kontrolle zum Schutz des Arbeitsplatzes und der Umwelt in der Produktion. Die ILMAC nimmt damit eine Herausforderung auf, welche mit der Diskussion um die Verantwortung der chemischen Industrie und der $\mathrm{Be}$ hörden verbunden ist.

Am begleitenden Kongress - dem 9. Basler Treffen für chemische Technik - finden zahlreiche Fachverbände den Rahmen zur Durchführung von Tagungen Wissenschaftler und Techniker aus aller Welt werden hier über den aktuellen Stand von Technik und Forschung berichten und diskutieren. Dem Besucher der ILMAC wird damit eine Bereicherung des Messebesuchs geboten.

\section{Vertreter der Schweiz wird Präsident des} europäischen Pharmaverbandes (EFPIA)

1.C. - Dr. Armin Kessler, Verwaltungsrat und Konzernleitungsmitglied der F. Hoffmann-La Roche AG, Basel, wurde anlässlich der EFPIA-Generalversammlung in Heidelberg einstimmig zum neuen Präsidenten für die Amtszeit 1990-92 gewählt. Kessler folgt auf $\mathrm{Dr}$ Alberto Aleotti (Menarini SpA) in einer Phase, die im Blick auf die Vollendung des EG-Binnenmarktes und die EWR-Verhandlungen für die pharmazeutische Industrie Europas von besonderer Bedeutung sein wird. Die EFPIA mit Sitz in Brüssel ist der europäische Pharmaverband, dem auch die Schweizerische Gesellschaft für Chemische Industrie (SGCI) als Vollmitglied angehört.

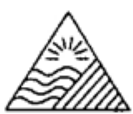

Internationa: Association of Environ mentel Analytical Chemistry Unter the patronage of the Swiss Feder

\section{2nd Soil Residue Analysis Workshop}

March 11-13, 1991

Ecole Polytechnique Fédérale de Lausanne (EPFL), Switzerland

Organization and information: Registration and Scientific Programme, Prof. Dr. Joseph Tarradellas, Chairman, IGE-EPFL, CH-1015 Lausanne, tel. (21) 693.27.12, fax. (21) 693.27.27
This Workshop is organized by the International Association of Environmental Analytical Chemistry and the Deparment of Analyticel Inorganic and Applied Chemistry of the University of Geneva. Swizertiand

Unter the Patronage of the Swiss Federal Otfice for the Environment, Forest and Landscape, Berne

\section{4th Hans Wolfgang Nürnberg \\ Memorial Workshop}

Toxic Metal Compounds

(Interrelation between Chemistry and Biology)

Congress-Center, CH-1865 Les Diablerets (Switzerland), March 4-8, 199

Organization and Information: Secretary and Address before and after the Workshop: Dr. Ernest Merian, Im Kirsgarten 22, CH-4106 Therwil (Switzerland), Tel. .. 4!-61-732950 (after 22nd April 1991 . 41-61-721 2950)

\section{Personalia}

\section{Geburtstage}

Alfred Hofstetter, Dr. sc. techn., Zürich, Mitglied des SChV, feiert am 3.10.90 seinen 60. Geburtstag.

Henri C. Silberman, Dr. chem., Laufen, Mitglied des SChV, feiert am 5.10 .90 seinen 65 . Geburtstag.

Rolf Schmid, Dr. Ing. Chem., Schwarzenburg, Mitglied des SChV, feiert am 6.10.90 seinen 65. Geburtstag.

Oswald Benz, Chemiker HTL, Grafenried, Mitglied des SChV, feiert am 11.10 .90 seinen 60 . Geburtstag.

Walter Frey, Dr. Ing. Chem., Muttenz, Mitglied des SChV, feiert am 12.10.90 seinen 75. Geburtstag.

Werner Manz, Dr. phil. II, Basel, Mitglied des SChV, feiert am 17.10.90 seinen 70. Geburtstag.

Ernst Kägi, Chemiker HTL, Monthey, Mitglied des SChV, feiert am 18.10 .90 seinen 60 . Geburtstag.

Max Sahli, Dr. phil., Chemiker, Kehrsatz, Mitglied des SChV, feiert am 23.10.90 seinen 65. Geburtstag.

Peter Jakober, Dr. sc. nat., Burgdorf, Mitglied des SChV, feiert am 24.10.90 seinen 60. Geburtstag.

Richard Neidlein, Prof. Dr., Heidelberg, Mitglied des SChV, feiert am 25.10 .90 seinen 60. Geburtstag.

U. Waltersperger, Chemiker HTL, Gossau, Mitglied des SChV, feiert am 29.10.90 seinen 65. Geburtstag.

\section{Neue Mitglieder}

Vogt-Schild AG, Druck und Verlag, Zuchwilerstrasse 21, 4501 Solothurn.

\section{Zusammenarbeit zwischen Roche und Western Capital für eine orale Form von Alpha-Interferon}

F. Hoffmann-La Roche AG, Basel, und die Western Capital Group (WCL) mit ihrer englischen PharmaTochtergesellschaft Cortecs Ltd. in London haben ein Abkommen über dic Zusammenarbeit zur Entwicklung und Prüfung einer oralen Form von alpha-Interferon unterzeichnet. Das Roche Präparat 'Roferon'-A wird hierbei zusammen mit dem Verabreichungssystem der WCL verwendet. Die Zusammenarbeit beruht auf früheren, von Cortecs durchgeführten klinischen Studien mit alpha-Interferon.

Interferone sind natürlich vorkommende Proteine oder Eiweisse. 'Roferon'-A ist das von Roche mittels Gentechnik produzierte Human-Interferon-alfa-2a; es ist in vielen Ländern als Mittel zur Behandlung von Haarzell-Leukämie, des durch AIDS verursachten Kaposi-Sarkoms und bösartiger Melanome zugelassen. Als weitere Indikationen werden derzeit registriert: chronische Hepatitis-B, kutanes T-Zell-Lymphom, chronisch-myeloische Leukämie und Nierenzellkrebs. Ausserdem wird 'Roferon'-A bei zusätzlichen Indikationen als Einzelsubstanz oder in Verbindung mit anderen Substanzen klinisch geprüft, so z. B. chronische Hepatitis-C, Darmkrebs sowie Lungenkrebs. Die Entwicklung einer oralen Form des Präparates könnte dic Behandlung dieser Krankheiten verbessern und den Alpha-Interferon-Markt erweitern.

In einer davon unabhängigen, zusätzlichen Entwicklung haben WCL und Cortecs im Mai 1990 bereits eine orale Form von Erythropoietin, dem kolonienstimulierenden Faktor roter Blutzellen, erfolgreich geprüft. Andere therapeutische Proteine, welche mit dem für grosse Moleküle bestimmten oralen Verabreichungssystem der WCL-Gruppe erfolgreich getestet wurden, schliessen Insulin, Calcitonin sowie menschliches Wachstumshormon ein.

\section{Fourth European Symposium on Radiopharmacy and Radiopharmaceuticals}

Baden (near Zürich), Switzerland Mai 1-4, 1991

Organized by the Radiopharmacy Group of the Swiss Society of Nuclear Medicine under the auspices of the EANM Taskgroup on Radiopharmaceuticals (Chairman: Prof. Dr. P. H. Cox)

The Fourth European Symposium will review recent developments in radiopharmacy and current research on radiopharmaceuticals. The scope will be expanded to include the pharmacokinetics and metabolism of radiopharmaceuticals, and the use of radioactive materials in drug formulation studies.

Secretary: c/o Radiopharmacy Division, Paul Scherrer Institutc, CH-5232 Villigen PSI, Switzerland, Telephone 0569928 14, Telex 827418 (psir ch), Telefax 056 982635 .

\section{EURO FOOD TOX III}

Schweizerische Chemische Gesellschaft Schweizerischer Chemiker-Verband

\section{Herbstversammlung in Bern}

Freitag, 19. Oktober 1990

Chemische Institute der Universität Bern, Freiestrasse 3

Programm $08.30-09.00$

09.00-09.30 Plenarvortrag des Werner-Preisträgers 1990 Dr. H. Frei, University of California Berkeley/USA

'Chemistry with red and near infrared light'

09.40-18.00 Kurzvorträge der Sektion für Organische Chemie

09.40-17.45 Vorträge der Sektion für Medizinische Chemie
09.40-15.40 Kurzvorträge der Sektion für Physikalische Chemie

14.20-17.00 Kurzvorträge der Sektion für Computerunterstützte Chemie

09.00-12.30 Minisymposium 'Recent Developments in Organometallic Chemistry and Homogeneous Catalysis'

14.30-16.30 Postersession der Sektion für Anorganische Chemie und Koordinationschemic

10.30-17.40 Seminar 'Analytische Chemie' 13.30-15.00 Postersession 'Analytische Chemie'

Information/Kontakt:

Dr. E. Zass, Sekretär SCG

ETH Zürich, Universitätstr. 16, CH-8092 Zürich
Interdisciplinary Conference on the Effects of Food on the Immune and Hormonal Systems sponsored by The European Society of Toxicology, The Federation of European Chemical Societies: Working Party on Food Chemistry, The Federation of European Nutrition Societies.

May 23-24, 1991, Zurich/Switzerland

Information: EURO FOOD TOX III, Sekretarial, MGB Zentrallabor, Postfach 266, 8031 Zürich, Switzerland.

\section{Der Schweizerische Schulrat}

hat den Ruzicka-Preis 1990 an Dr. Charles Fehr, Firmenich SA, Research Laboratories, Genf, verliehen. Anlässlich der Preisverleihung vom 10. September 1990 an der ETH Zürich hat der Preisträger folgenden Vortrag gehalten: Enantioselektive Protonierung von Enolaten zur Synthese von Naturstoffen. 
band für Umwelttechnik und dem Schweizerischen Elektrotechnischen Komitee (CES) Fachkommission 31 realisiert. Gezeigt werden neue Produkte und Technologien für den Einsatz elektrischer Betriebsmittel in explosionsgefährdeten Bereichen. Während der Dauer der Sonderschau stehen den Besuchern Mitglieder des Eidg. Starkstrominspektorates, des SEV (Schweizerischer Elektrotechnischer Verein), der SUVA und der Fachgruppe 31 für Fachgespräche und Auskünfte zur Verfügung.

Ein Hauptanliegen der Messe ist das Aufzeigen neuer Möglichkeiten und verbesserter Technologien zum Schutz von Mensch und Umwelt. In diese Richtung zielt nicht nur die Sonderschau. Das Thema der Messe verweist generell darauf, schaffen doch neue Werkstoffe wie auch neue Technologien die Grundlage für verfeinerte Methoden der Messung und der Kontrolle zum Schutz des Arbeitsplatzes und der Umwelt in der Produktion. Die ILMAC nimmt damit eine Herausforderung auf, welche mit der Diskussion um die Verantwortung der chemischen Industrie und der $\mathrm{Be}$ hörden verbunden ist.

Am begleitenden Kongress - dem 9. Basler Treffen für chemische Technik - finden zahlreiche Fachverbände den Rahmen zur Durchführung von Tagungen Wissenschaftler und Techniker aus aller Welt werden hier über den aktuellen Stand von Technik und Forschung berichten und diskutieren. Dem Besucher der ILMAC wird damit eine Bereicherung des Messebesuchs geboten.

\section{Vertreter der Schweiz wird Präsident des} europäischen Pharmaverbandes (EFPIA)

1.C. - Dr. Armin Kessler, Verwaltungsrat und Konzernleitungsmitglied der F. Hoffmann-La Roche AG, Basel, wurde anlässlich der EFPIA-Generalversammlung in Heidelberg einstimmig zum neuen Präsidenten für die Amtszeit 1990-92 gewählt. Kessler folgt auf $\mathrm{Dr}$ Alberto Aleotti (Menarini SpA) in einer Phase, die im Blick auf die Vollendung des EG-Binnenmarktes und die EWR-Verhandlungen für die pharmazeutische Industrie Europas von besonderer Bedeutung sein wird. Die EFPIA mit Sitz in Brüssel ist der europäische Pharmaverband, dem auch die Schweizerische Gesellschaft für Chemische Industrie (SGCI) als Vollmitglied angehört.

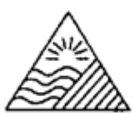

Internationa: Association of Environ mentel Analytical Chemistry Unter the patronage of the Swiss Feder

\section{2nd Soil Residue Analysis Workshop}

March 11-13, 1991

Ecole Polytechnique Fédérale de Lausanne (EPFL), Switzerland

Organization and information: Registration and Scientific Programme, Prof. Dr. Joseph Tarradellas, Chairman, IGE-EPFL, CH-1015 Lausanne, tel. (21) 693.27.12, fax. (21) 693.27.27
This Workshop is organized by the International Association of Environmental Analytical Chemistry and the Deparment of Analyticel Inorganic and Applied Chemistry of the University of Geneva. Swizertiand

Unter the Patronage of the Swiss Federal Otfice for the Environment, Forest and Landscape, Berne

\section{4th Hans Wolfgang Nürnberg \\ Memorial Workshop}

Toxic Metal Compounds

(Interrelation between Chemistry and Biology)

Congress-Center, CH-1865 Les Diablerets (Switzerland), March 4-8, 199

Organization and Information: Secretary and Address before and after the Workshop: Dr. Ernest Merian, Im Kirsgarten 22, CH-4106 Therwil (Switzerland), Tel. .. 4!-61-732950 (after 22nd April 1991 . 41-61-721 2950)

\section{Personalia}

\section{Geburtstage}

Alfred Hofstetter, Dr. sc. techn., Zürich, Mitglied des SChV, feiert am 3.10.90 seinen 60. Geburtstag.

Henri C. Silberman, Dr. chem., Laufen, Mitglied des SChV, feiert am 5.10 .90 seinen 65 . Geburtstag.

Rolf Schmid, Dr. Ing. Chem., Schwarzenburg, Mitglied des SChV, feiert am 6.10.90 seinen 65. Geburtstag.

Oswald Benz, Chemiker HTL, Grafenried, Mitglied des SChV, feiert am 11.10 .90 seinen 60 . Geburtstag.

Walter Frey, Dr. Ing. Chem., Muttenz, Mitglied des SChV, feiert am 12.10.90 seinen 75. Geburtstag.

Werner Manz, Dr. phil. II, Basel, Mitglied des SChV, feiert am 17.10.90 seinen 70. Geburtstag.

Ernst Kägi, Chemiker HTL, Monthey, Mitglied des SChV, feiert am 18.10 .90 seinen 60 . Geburtstag.

Max Sahli, Dr. phil., Chemiker, Kehrsatz, Mitglied des SChV, feiert am 23.10.90 seinen 65. Geburtstag.

Peter Jakober, Dr. sc. nat., Burgdorf, Mitglied des SChV, feiert am 24.10.90 seinen 60. Geburtstag.

Richard Neidlein, Prof. Dr., Heidelberg, Mitglied des SChV, feiert am 25.10 .90 seinen 60. Geburtstag.

U. Waltersperger, Chemiker HTL, Gossau, Mitglied des SChV, feiert am 29.10.90 seinen 65. Geburtstag.

\section{Neue Mitglieder}

Vogt-Schild AG, Druck und Verlag, Zuchwilerstrasse 21, 4501 Solothurn.

\section{Zusammenarbeit zwischen Roche und Western Capital für eine orale Form von Alpha-Interferon}

F. Hoffmann-La Roche AG, Basel, und die Western Capital Group (WCL) mit ihrer englischen PharmaTochtergesellschaft Cortecs Ltd. in London haben ein Abkommen über dic Zusammenarbeit zur Entwicklung und Prüfung einer oralen Form von alpha-Interferon unterzeichnet. Das Roche Präparat 'Roferon'-A wird hierbei zusammen mit dem Verabreichungssystem der WCL verwendet. Die Zusammenarbeit beruht auf früheren, von Cortecs durchgeführten klinischen Studien mit alpha-Interferon.

Interferone sind natürlich vorkommende Proteine oder Eiweisse. 'Roferon'-A ist das von Roche mittels Gentechnik produzierte Human-Interferon-alfa-2a; es ist in vielen Ländern als Mittel zur Behandlung von Haarzell-Leukämie, des durch AIDS verursachten Kaposi-Sarkoms und bösartiger Melanome zugelassen. Als weitere Indikationen werden derzeit registriert: chronische Hepatitis-B, kutanes T-Zell-Lymphom, chronisch-myeloische Leukämie und Nierenzellkrebs. Ausserdem wird 'Roferon'-A bei zusätzlichen Indikationen als Einzelsubstanz oder in Verbindung mit anderen Substanzen klinisch geprüft, so z. B. chronische Hepatitis-C, Darmkrebs sowie Lungenkrebs. Die Entwicklung einer oralen Form des Präparates könnte dic Behandlung dieser Krankheiten verbessern und den Alpha-Interferon-Markt erweitern.

In einer davon unabhängigen, zusätzlichen Entwicklung haben WCL und Cortecs im Mai 1990 bereits eine orale Form von Erythropoietin, dem kolonienstimulierenden Faktor roter Blutzellen, erfolgreich geprüft. Andere therapeutische Proteine, welche mit dem für grosse Moleküle bestimmten oralen Verabreichungssystem der WCL-Gruppe erfolgreich getestet wurden, schliessen Insulin, Calcitonin sowie menschliches Wachstumshormon ein.

\section{Fourth European Symposium on Radiopharmacy and Radiopharmaceuticals}

Baden (near Zürich), Switzerland Mai 1-4, 1991

Organized by the Radiopharmacy Group of the Swiss Society of Nuclear Medicine under the auspices of the EANM Taskgroup on Radiopharmaceuticals (Chairman: Prof. Dr. P. H. Cox)

The Fourth European Symposium will review recent developments in radiopharmacy and current research on radiopharmaceuticals. The scope will be expanded to include the pharmacokinetics and metabolism of radiopharmaceuticals, and the use of radioactive materials in drug formulation studies.

Secretary: c/o Radiopharmacy Division, Paul Scherrer Institutc, CH-5232 Villigen PSI, Switzerland, Telephone 0569928 14, Telex 827418 (psir ch), Telefax 056 982635 .

\section{EURO FOOD TOX III}

Schweizerische Chemische Gesellschaft Schweizerischer Chemiker-Verband

\section{Herbstversammlung in Bern}

Freitag, 19. Oktober 1990

Chemische Institute der Universität Bern, Freiestrasse 3

Programm $08.30-09.00$

09.00-09.30 Plenarvortrag des Werner-Preisträgers 1990 Dr. H. Frei, University of California Berkeley/USA

'Chemistry with red and near infrared light'

09.40-18.00 Kurzvorträge der Sektion für Organische Chemie

09.40-17.45 Vorträge der Sektion für Medizinische Chemie
09.40-15.40 Kurzvorträge der Sektion für Physikalische Chemie

14.20-17.00 Kurzvorträge der Sektion für Computerunterstützte Chemie

09.00-12.30 Minisymposium 'Recent Developments in Organometallic Chemistry and Homogeneous Catalysis'

14.30-16.30 Postersession der Sektion für Anorganische Chemie und Koordinationschemic

10.30-17.40 Seminar 'Analytische Chemie' 13.30-15.00 Postersession 'Analytische Chemie'

Information/Kontakt:

Dr. E. Zass, Sekretär SCG

ETH Zürich, Universitätstr. 16, CH-8092 Zürich
Interdisciplinary Conference on the Effects of Food on the Immune and Hormonal Systems sponsored by The European Society of Toxicology, The Federation of European Chemical Societies: Working Party on Food Chemistry, The Federation of European Nutrition Societies.

May 23-24, 1991, Zurich/Switzerland

Information: EURO FOOD TOX III, Sekretarial, MGB Zentrallabor, Postfach 266, 8031 Zürich, Switzerland.

\section{Der Schweizerische Schulrat}

hat den Ruzicka-Preis 1990 an Dr. Charles Fehr, Firmenich SA, Research Laboratories, Genf, verliehen. Anlässlich der Preisverleihung vom 10. September 1990 an der ETH Zürich hat der Preisträger folgenden Vortrag gehalten: Enantioselektive Protonierung von Enolaten zur Synthese von Naturstoffen. 
band für Umwelttechnik und dem Schweizerischen Elektrotechnischen Komitee (CES) Fachkommission 31 realisiert. Gezeigt werden neue Produkte und Technologien für den Einsatz elektrischer Betriebsmittel in explosionsgefährdeten Bereichen. Während der Dauer der Sonderschau stehen den Besuchern Mitglieder des Eidg. Starkstrominspektorates, des SEV (Schweizerischer Elektrotechnischer Verein), der SUVA und der Fachgruppe 31 für Fachgespräche und Auskünfte zur Verfügung.

Ein Hauptanliegen der Messe ist das Aufzeigen neuer Möglichkeiten und verbesserter Technologien zum Schutz von Mensch und Umwelt. In diese Richtung zielt nicht nur die Sonderschau. Das Thema der Messe verweist generell darauf, schaffen doch neue Werkstoffe wie auch neue Technologien die Grundlage für verfeinerte Methoden der Messung und der Kontrolle zum Schutz des Arbeitsplatzes und der Umwelt in der Produktion. Die ILMAC nimmt damit eine Herausforderung auf, welche mit der Diskussion um die Verantwortung der chemischen Industrie und der $\mathrm{Be}$ hörden verbunden ist.

Am begleitenden Kongress - dem 9. Basler Treffen für chemische Technik - finden zahlreiche Fachverbände den Rahmen zur Durchführung von Tagungen Wissenschaftler und Techniker aus aller Welt werden hier über den aktuellen Stand von Technik und Forschung berichten und diskutieren. Dem Besucher der ILMAC wird damit eine Bereicherung des Messebesuchs geboten.

\section{Vertreter der Schweiz wird Präsident des} europäischen Pharmaverbandes (EFPIA)

1.C. - Dr. Armin Kessler, Verwaltungsrat und Konzernleitungsmitglied der F. Hoffmann-La Roche AG, Basel, wurde anlässlich der EFPIA-Generalversammlung in Heidelberg einstimmig zum neuen Präsidenten für die Amtszeit 1990-92 gewählt. Kessler folgt auf $\mathrm{Dr}$ Alberto Aleotti (Menarini SpA) in einer Phase, die im Blick auf die Vollendung des EG-Binnenmarktes und die EWR-Verhandlungen für die pharmazeutische Industrie Europas von besonderer Bedeutung sein wird. Die EFPIA mit Sitz in Brüssel ist der europäische Pharmaverband, dem auch die Schweizerische Gesellschaft für Chemische Industrie (SGCI) als Vollmitglied angehört.

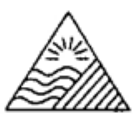

Internationa: Association of Environ mentel Analytical Chemistry Unter the patronage of the Swiss Feder

\section{2nd Soil Residue Analysis Workshop}

March 11-13, 1991

Ecole Polytechnique Fédérale de Lausanne (EPFL), Switzerland

Organization and information: Registration and Scientific Programme, Prof. Dr. Joseph Tarradellas, Chairman, IGE-EPFL, CH-1015 Lausanne, tel. (21) 693.27.12, fax. (21) 693.27.27
This Workshop is organized by the International Association of Environmental Analytical Chemistry and the Deparment of Analyticel Inorganic and Applied Chemistry of the University of Geneva. Swizertiand

Unter the Patronage of the Swiss Federal Otfice for the Environment, Forest and Landscape, Berne

\section{4th Hans Wolfgang Nürnberg \\ Memorial Workshop}

Toxic Metal Compounds

(Interrelation between Chemistry and Biology)

Congress-Center, CH-1865 Les Diablerets (Switzerland), March 4-8, 199

Organization and Information: Secretary and Address before and after the Workshop: Dr. Ernest Merian, Im Kirsgarten 22, CH-4106 Therwil (Switzerland), Tel. .. 4!-61-732950 (after 22nd April 1991 . 41-61-721 2950)

\section{Personalia}

\section{Geburtstage}

Alfred Hofstetter, Dr. sc. techn., Zürich, Mitglied des SChV, feiert am 3.10.90 seinen 60. Geburtstag.

Henri C. Silberman, Dr. chem., Laufen, Mitglied des SChV, feiert am 5.10 .90 seinen 65 . Geburtstag.

Rolf Schmid, Dr. Ing. Chem., Schwarzenburg, Mitglied des SChV, feiert am 6.10.90 seinen 65. Geburtstag.

Oswald Benz, Chemiker HTL, Grafenried, Mitglied des SChV, feiert am 11.10 .90 seinen 60 . Geburtstag.

Walter Frey, Dr. Ing. Chem., Muttenz, Mitglied des SChV, feiert am 12.10.90 seinen 75. Geburtstag.

Werner Manz, Dr. phil. II, Basel, Mitglied des SChV, feiert am 17.10.90 seinen 70. Geburtstag.

Ernst Kägi, Chemiker HTL, Monthey, Mitglied des SChV, feiert am 18.10 .90 seinen 60 . Geburtstag.

Max Sahli, Dr. phil., Chemiker, Kehrsatz, Mitglied des SChV, feiert am 23.10.90 seinen 65. Geburtstag.

Peter Jakober, Dr. sc. nat., Burgdorf, Mitglied des SChV, feiert am 24.10.90 seinen 60. Geburtstag.

Richard Neidlein, Prof. Dr., Heidelberg, Mitglied des SChV, feiert am 25.10 .90 seinen 60. Geburtstag.

U. Waltersperger, Chemiker HTL, Gossau, Mitglied des SChV, feiert am 29.10.90 seinen 65. Geburtstag.

\section{Neue Mitglieder}

Vogt-Schild AG, Druck und Verlag, Zuchwilerstrasse 21, 4501 Solothurn.

\section{Zusammenarbeit zwischen Roche und Western Capital für eine orale Form von Alpha-Interferon}

F. Hoffmann-La Roche AG, Basel, und die Western Capital Group (WCL) mit ihrer englischen PharmaTochtergesellschaft Cortecs Ltd. in London haben ein Abkommen über dic Zusammenarbeit zur Entwicklung und Prüfung einer oralen Form von alpha-Interferon unterzeichnet. Das Roche Präparat 'Roferon'-A wird hierbei zusammen mit dem Verabreichungssystem der WCL verwendet. Die Zusammenarbeit beruht auf früheren, von Cortecs durchgeführten klinischen Studien mit alpha-Interferon.

Interferone sind natürlich vorkommende Proteine oder Eiweisse. 'Roferon'-A ist das von Roche mittels Gentechnik produzierte Human-Interferon-alfa-2a; es ist in vielen Ländern als Mittel zur Behandlung von Haarzell-Leukämie, des durch AIDS verursachten Kaposi-Sarkoms und bösartiger Melanome zugelassen. Als weitere Indikationen werden derzeit registriert: chronische Hepatitis-B, kutanes T-Zell-Lymphom, chronisch-myeloische Leukämie und Nierenzellkrebs. Ausserdem wird 'Roferon'-A bei zusätzlichen Indikationen als Einzelsubstanz oder in Verbindung mit anderen Substanzen klinisch geprüft, so z. B. chronische Hepatitis-C, Darmkrebs sowie Lungenkrebs. Die Entwicklung einer oralen Form des Präparates könnte dic Behandlung dieser Krankheiten verbessern und den Alpha-Interferon-Markt erweitern.

In einer davon unabhängigen, zusätzlichen Entwicklung haben WCL und Cortecs im Mai 1990 bereits eine orale Form von Erythropoietin, dem kolonienstimulierenden Faktor roter Blutzellen, erfolgreich geprüft. Andere therapeutische Proteine, welche mit dem für grosse Moleküle bestimmten oralen Verabreichungssystem der WCL-Gruppe erfolgreich getestet wurden, schliessen Insulin, Calcitonin sowie menschliches Wachstumshormon ein.

\section{Fourth European Symposium on Radiopharmacy and Radiopharmaceuticals}

Baden (near Zürich), Switzerland Mai 1-4, 1991

Organized by the Radiopharmacy Group of the Swiss Society of Nuclear Medicine under the auspices of the EANM Taskgroup on Radiopharmaceuticals (Chairman: Prof. Dr. P. H. Cox)

The Fourth European Symposium will review recent developments in radiopharmacy and current research on radiopharmaceuticals. The scope will be expanded to include the pharmacokinetics and metabolism of radiopharmaceuticals, and the use of radioactive materials in drug formulation studies.

Secretary: c/o Radiopharmacy Division, Paul Scherrer Institutc, CH-5232 Villigen PSI, Switzerland, Telephone 0569928 14, Telex 827418 (psir ch), Telefax 056 982635 .

\section{EURO FOOD TOX III}

Schweizerische Chemische Gesellschaft Schweizerischer Chemiker-Verband

\section{Herbstversammlung in Bern}

Freitag, 19. Oktober 1990

Chemische Institute der Universität Bern, Freiestrasse 3

Programm $08.30-09.00$

09.00-09.30 Plenarvortrag des Werner-Preisträgers 1990 Dr. H. Frei, University of California Berkeley/USA

'Chemistry with red and near infrared light'

09.40-18.00 Kurzvorträge der Sektion für Organische Chemie

09.40-17.45 Vorträge der Sektion für Medizinische Chemie
09.40-15.40 Kurzvorträge der Sektion für Physikalische Chemie

14.20-17.00 Kurzvorträge der Sektion für Computerunterstützte Chemie

09.00-12.30 Minisymposium 'Recent Developments in Organometallic Chemistry and Homogeneous Catalysis'

14.30-16.30 Postersession der Sektion für Anorganische Chemie und Koordinationschemic

10.30-17.40 Seminar 'Analytische Chemie' 13.30-15.00 Postersession 'Analytische Chemie'

Information/Kontakt:

Dr. E. Zass, Sekretär SCG

ETH Zürich, Universitätstr. 16, CH-8092 Zürich
Interdisciplinary Conference on the Effects of Food on the Immune and Hormonal Systems sponsored by The European Society of Toxicology, The Federation of European Chemical Societies: Working Party on Food Chemistry, The Federation of European Nutrition Societies.

May 23-24, 1991, Zurich/Switzerland

Information: EURO FOOD TOX III, Sekretarial, MGB Zentrallabor, Postfach 266, 8031 Zürich, Switzerland.

\section{Der Schweizerische Schulrat}

hat den Ruzicka-Preis 1990 an Dr. Charles Fehr, Firmenich SA, Research Laboratories, Genf, verliehen. Anlässlich der Preisverleihung vom 10. September 1990 an der ETH Zürich hat der Preisträger folgenden Vortrag gehalten: Enantioselektive Protonierung von Enolaten zur Synthese von Naturstoffen. 
band für Umwelttechnik und dem Schweizerischen Elektrotechnischen Komitee (CES) Fachkommission 31 realisiert. Gezeigt werden neue Produkte und Technologien für den Einsatz elektrischer Betriebsmittel in explosionsgefährdeten Bereichen. Während der Dauer der Sonderschau stehen den Besuchern Mitglieder des Eidg. Starkstrominspektorates, des SEV (Schweizerischer Elektrotechnischer Verein), der SUVA und der Fachgruppe 31 für Fachgespräche und Auskünfte zur Verfügung.

Ein Hauptanliegen der Messe ist das Aufzeigen neuer Möglichkeiten und verbesserter Technologien zum Schutz von Mensch und Umwelt. In diese Richtung zielt nicht nur die Sonderschau. Das Thema der Messe verweist generell darauf, schaffen doch neue Werkstoffe wie auch neue Technologien die Grundlage für verfeinerte Methoden der Messung und der Kontrolle zum Schutz des Arbeitsplatzes und der Umwelt in der Produktion. Die ILMAC nimmt damit eine Herausforderung auf, welche mit der Diskussion um die Verantwortung der chemischen Industrie und der $\mathrm{Be}$ hörden verbunden ist.

Am begleitenden Kongress - dem 9. Basler Treffen für chemische Technik - finden zahlreiche Fachverbände den Rahmen zur Durchführung von Tagungen Wissenschaftler und Techniker aus aller Welt werden hier über den aktuellen Stand von Technik und Forschung berichten und diskutieren. Dem Besucher der ILMAC wird damit eine Bereicherung des Messebesuchs geboten.

\section{Vertreter der Schweiz wird Präsident des} europäischen Pharmaverbandes (EFPIA)

1.C. - Dr. Armin Kessler, Verwaltungsrat und Konzernleitungsmitglied der F. Hoffmann-La Roche AG, Basel, wurde anlässlich der EFPIA-Generalversammlung in Heidelberg einstimmig zum neuen Präsidenten für die Amtszeit 1990-92 gewählt. Kessler folgt auf $\mathrm{Dr}$ Alberto Aleotti (Menarini SpA) in einer Phase, die im Blick auf die Vollendung des EG-Binnenmarktes und die EWR-Verhandlungen für die pharmazeutische Industrie Europas von besonderer Bedeutung sein wird. Die EFPIA mit Sitz in Brüssel ist der europäische Pharmaverband, dem auch die Schweizerische Gesellschaft für Chemische Industrie (SGCI) als Vollmitglied angehört.

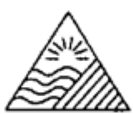

Internationa: Association of Environ mentel Analytical Chemistry Unter the patronage of the Swiss Feder

\section{2nd Soil Residue Analysis Workshop}

March 11-13, 1991

Ecole Polytechnique Fédérale de Lausanne (EPFL), Switzerland

Organization and information: Registration and Scientific Programme, Prof. Dr. Joseph Tarradellas, Chairman, IGE-EPFL, CH-1015 Lausanne, tel. (21) 693.27.12, fax. (21) 693.27.27
This Workshop is organized by the International Association of Environmental Analytical Chemistry and the Deparment of Analyticel Inorganic and Applied Chemistry of the University of Geneva. Swizertiand

Unter the Patronage of the Swiss Federal Otfice for the Environment, Forest and Landscape, Berne

\section{4th Hans Wolfgang Nürnberg \\ Memorial Workshop}

Toxic Metal Compounds

(Interrelation between Chemistry and Biology)

Congress-Center, CH-1865 Les Diablerets (Switzerland), March 4-8, 199

Organization and Information: Secretary and Address before and after the Workshop: Dr. Ernest Merian, Im Kirsgarten 22, CH-4106 Therwil (Switzerland), Tel. .. 4!-61-732950 (after 22nd April 1991 . 41-61-721 2950)

\section{Personalia}

\section{Geburtstage}

Alfred Hofstetter, Dr. sc. techn., Zürich, Mitglied des SChV, feiert am 3.10.90 seinen 60. Geburtstag.

Henri C. Silberman, Dr. chem., Laufen, Mitglied des SChV, feiert am 5.10 .90 seinen 65 . Geburtstag.

Rolf Schmid, Dr. Ing. Chem., Schwarzenburg, Mitglied des SChV, feiert am 6.10.90 seinen 65. Geburtstag.

Oswald Benz, Chemiker HTL, Grafenried, Mitglied des SChV, feiert am 11.10 .90 seinen 60 . Geburtstag.

Walter Frey, Dr. Ing. Chem., Muttenz, Mitglied des SChV, feiert am 12.10.90 seinen 75. Geburtstag.

Werner Manz, Dr. phil. II, Basel, Mitglied des SChV, feiert am 17.10.90 seinen 70. Geburtstag.

Ernst Kägi, Chemiker HTL, Monthey, Mitglied des SChV, feiert am 18.10 .90 seinen 60 . Geburtstag.

Max Sahli, Dr. phil., Chemiker, Kehrsatz, Mitglied des SChV, feiert am 23.10.90 seinen 65. Geburtstag.

Peter Jakober, Dr. sc. nat., Burgdorf, Mitglied des SChV, feiert am 24.10.90 seinen 60. Geburtstag.

Richard Neidlein, Prof. Dr., Heidelberg, Mitglied des SChV, feiert am 25.10 .90 seinen 60. Geburtstag.

U. Waltersperger, Chemiker HTL, Gossau, Mitglied des SChV, feiert am 29.10.90 seinen 65. Geburtstag.

\section{Neue Mitglieder}

Vogt-Schild AG, Druck und Verlag, Zuchwilerstrasse 21, 4501 Solothurn.

\section{Zusammenarbeit zwischen Roche und Western Capital für eine orale Form von Alpha-Interferon}

F. Hoffmann-La Roche AG, Basel, und die Western Capital Group (WCL) mit ihrer englischen PharmaTochtergesellschaft Cortecs Ltd. in London haben ein Abkommen über dic Zusammenarbeit zur Entwicklung und Prüfung einer oralen Form von alpha-Interferon unterzeichnet. Das Roche Präparat 'Roferon'-A wird hierbei zusammen mit dem Verabreichungssystem der WCL verwendet. Die Zusammenarbeit beruht auf früheren, von Cortecs durchgeführten klinischen Studien mit alpha-Interferon.

Interferone sind natürlich vorkommende Proteine oder Eiweisse. 'Roferon'-A ist das von Roche mittels Gentechnik produzierte Human-Interferon-alfa-2a; es ist in vielen Ländern als Mittel zur Behandlung von Haarzell-Leukämie, des durch AIDS verursachten Kaposi-Sarkoms und bösartiger Melanome zugelassen. Als weitere Indikationen werden derzeit registriert: chronische Hepatitis-B, kutanes T-Zell-Lymphom, chronisch-myeloische Leukämie und Nierenzellkrebs. Ausserdem wird 'Roferon'-A bei zusätzlichen Indikationen als Einzelsubstanz oder in Verbindung mit anderen Substanzen klinisch geprüft, so z. B. chronische Hepatitis-C, Darmkrebs sowie Lungenkrebs. Die Entwicklung einer oralen Form des Präparates könnte dic Behandlung dieser Krankheiten verbessern und den Alpha-Interferon-Markt erweitern.

In einer davon unabhängigen, zusätzlichen Entwicklung haben WCL und Cortecs im Mai 1990 bereits eine orale Form von Erythropoietin, dem kolonienstimulierenden Faktor roter Blutzellen, erfolgreich geprüft. Andere therapeutische Proteine, welche mit dem für grosse Moleküle bestimmten oralen Verabreichungssystem der WCL-Gruppe erfolgreich getestet wurden, schliessen Insulin, Calcitonin sowie menschliches Wachstumshormon ein.

\section{Fourth European Symposium on Radiopharmacy and Radiopharmaceuticals}

Baden (near Zürich), Switzerland Mai 1-4, 1991

Organized by the Radiopharmacy Group of the Swiss Society of Nuclear Medicine under the auspices of the EANM Taskgroup on Radiopharmaceuticals (Chairman: Prof. Dr. P. H. Cox)

The Fourth European Symposium will review recent developments in radiopharmacy and current research on radiopharmaceuticals. The scope will be expanded to include the pharmacokinetics and metabolism of radiopharmaceuticals, and the use of radioactive materials in drug formulation studies.

Secretary: c/o Radiopharmacy Division, Paul Scherrer Institutc, CH-5232 Villigen PSI, Switzerland, Telephone 0569928 14, Telex 827418 (psir ch), Telefax 056 982635 .

\section{EURO FOOD TOX III}

Schweizerische Chemische Gesellschaft Schweizerischer Chemiker-Verband

\section{Herbstversammlung in Bern}

Freitag, 19. Oktober 1990

Chemische Institute der Universität Bern, Freiestrasse 3

Programm $08.30-09.00$

09.00-09.30 Plenarvortrag des Werner-Preisträgers 1990 Dr. H. Frei, University of California Berkeley/USA

'Chemistry with red and near infrared light'

09.40-18.00 Kurzvorträge der Sektion für Organische Chemie

09.40-17.45 Vorträge der Sektion für Medizinische Chemie
09.40-15.40 Kurzvorträge der Sektion für Physikalische Chemie

14.20-17.00 Kurzvorträge der Sektion für Computerunterstützte Chemie

09.00-12.30 Minisymposium 'Recent Developments in Organometallic Chemistry and Homogeneous Catalysis'

14.30-16.30 Postersession der Sektion für Anorganische Chemie und Koordinationschemic

10.30-17.40 Seminar 'Analytische Chemie' 13.30-15.00 Postersession 'Analytische Chemie'

Information/Kontakt:

Dr. E. Zass, Sekretär SCG

ETH Zürich, Universitätstr. 16, CH-8092 Zürich
Interdisciplinary Conference on the Effects of Food on the Immune and Hormonal Systems sponsored by The European Society of Toxicology, The Federation of European Chemical Societies: Working Party on Food Chemistry, The Federation of European Nutrition Societies.

May 23-24, 1991, Zurich/Switzerland

Information: EURO FOOD TOX III, Sekretarial, MGB Zentrallabor, Postfach 266, 8031 Zürich, Switzerland.

\section{Der Schweizerische Schulrat}

hat den Ruzicka-Preis 1990 an Dr. Charles Fehr, Firmenich SA, Research Laboratories, Genf, verliehen. Anlässlich der Preisverleihung vom 10. September 1990 an der ETH Zürich hat der Preisträger folgenden Vortrag gehalten: Enantioselektive Protonierung von Enolaten zur Synthese von Naturstoffen. 
band für Umwelttechnik und dem Schweizerischen Elektrotechnischen Komitee (CES) Fachkommission 31 realisiert. Gezeigt werden neue Produkte und Technologien für den Einsatz elektrischer Betriebsmittel in explosionsgefährdeten Bereichen. Während der Dauer der Sonderschau stehen den Besuchern Mitglieder des Eidg. Starkstrominspektorates, des SEV (Schweizerischer Elektrotechnischer Verein), der SUVA und der Fachgruppe 31 für Fachgespräche und Auskünfte zur Verfügung.

Ein Hauptanliegen der Messe ist das Aufzeigen neuer Möglichkeiten und verbesserter Technologien zum Schutz von Mensch und Umwelt. In diese Richtung zielt nicht nur die Sonderschau. Das Thema der Messe verweist generell darauf, schaffen doch neue Werkstoffe wie auch neue Technologien die Grundlage für verfeinerte Methoden der Messung und der Kontrolle zum Schutz des Arbeitsplatzes und der Umwelt in der Produktion. Die ILMAC nimmt damit eine Herausforderung auf, welche mit der Diskussion um die Verantwortung der chemischen Industrie und der $\mathrm{Be}$ hörden verbunden ist.

Am begleitenden Kongress - dem 9. Basler Treffen für chemische Technik - finden zahlreiche Fachverbände den Rahmen zur Durchführung von Tagungen Wissenschaftler und Techniker aus aller Welt werden hier über den aktuellen Stand von Technik und Forschung berichten und diskutieren. Dem Besucher der ILMAC wird damit eine Bereicherung des Messebesuchs geboten.

\section{Vertreter der Schweiz wird Präsident des} europäischen Pharmaverbandes (EFPIA)

1.C. - Dr. Armin Kessler, Verwaltungsrat und Konzernleitungsmitglied der F. Hoffmann-La Roche AG, Basel, wurde anlässlich der EFPIA-Generalversammlung in Heidelberg einstimmig zum neuen Präsidenten für die Amtszeit 1990-92 gewählt. Kessler folgt auf $\mathrm{Dr}$ Alberto Aleotti (Menarini SpA) in einer Phase, die im Blick auf die Vollendung des EG-Binnenmarktes und die EWR-Verhandlungen für die pharmazeutische Industrie Europas von besonderer Bedeutung sein wird. Die EFPIA mit Sitz in Brüssel ist der europäische Pharmaverband, dem auch die Schweizerische Gesellschaft für Chemische Industrie (SGCI) als Vollmitglied angehört.

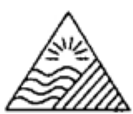

Internationa: Association of Environ mentel Analytical Chemistry Unter the patronage of the Swiss Feder

\section{2nd Soil Residue Analysis Workshop}

March 11-13, 1991

Ecole Polytechnique Fédérale de Lausanne (EPFL), Switzerland

Organization and information: Registration and Scientific Programme, Prof. Dr. Joseph Tarradellas, Chairman, IGE-EPFL, CH-1015 Lausanne, tel. (21) 693.27.12, fax. (21) 693.27.27
This Workshop is organized by the International Association of Environmental Analytical Chemistry and the Deparment of Analyticel Inorganic and Applied Chemistry of the University of Geneva. Swizertiand

Unter the Patronage of the Swiss Federal Otfice for the Environment, Forest and Landscape, Berne

\section{4th Hans Wolfgang Nürnberg \\ Memorial Workshop}

Toxic Metal Compounds

(Interrelation between Chemistry and Biology)

Congress-Center, CH-1865 Les Diablerets (Switzerland), March 4-8, 199

Organization and Information: Secretary and Address before and after the Workshop: Dr. Ernest Merian, Im Kirsgarten 22, CH-4106 Therwil (Switzerland), Tel. .. 4!-61-732950 (after 22nd April 1991 . 41-61-721 2950)

\section{Personalia}

\section{Geburtstage}

Alfred Hofstetter, Dr. sc. techn., Zürich, Mitglied des SChV, feiert am 3.10.90 seinen 60. Geburtstag.

Henri C. Silberman, Dr. chem., Laufen, Mitglied des SChV, feiert am 5.10 .90 seinen 65 . Geburtstag.

Rolf Schmid, Dr. Ing. Chem., Schwarzenburg, Mitglied des SChV, feiert am 6.10.90 seinen 65. Geburtstag.

Oswald Benz, Chemiker HTL, Grafenried, Mitglied des SChV, feiert am 11.10 .90 seinen 60 . Geburtstag.

Walter Frey, Dr. Ing. Chem., Muttenz, Mitglied des SChV, feiert am 12.10.90 seinen 75. Geburtstag.

Werner Manz, Dr. phil. II, Basel, Mitglied des SChV, feiert am 17.10.90 seinen 70. Geburtstag.

Ernst Kägi, Chemiker HTL, Monthey, Mitglied des SChV, feiert am 18.10 .90 seinen 60 . Geburtstag.

Max Sahli, Dr. phil., Chemiker, Kehrsatz, Mitglied des SChV, feiert am 23.10.90 seinen 65. Geburtstag.

Peter Jakober, Dr. sc. nat., Burgdorf, Mitglied des SChV, feiert am 24.10.90 seinen 60. Geburtstag.

Richard Neidlein, Prof. Dr., Heidelberg, Mitglied des SChV, feiert am 25.10 .90 seinen 60. Geburtstag.

U. Waltersperger, Chemiker HTL, Gossau, Mitglied des SChV, feiert am 29.10.90 seinen 65. Geburtstag.

\section{Neue Mitglieder}

Vogt-Schild AG, Druck und Verlag, Zuchwilerstrasse 21, 4501 Solothurn.

\section{Zusammenarbeit zwischen Roche und Western Capital für eine orale Form von Alpha-Interferon}

F. Hoffmann-La Roche AG, Basel, und die Western Capital Group (WCL) mit ihrer englischen PharmaTochtergesellschaft Cortecs Ltd. in London haben ein Abkommen über dic Zusammenarbeit zur Entwicklung und Prüfung einer oralen Form von alpha-Interferon unterzeichnet. Das Roche Präparat 'Roferon'-A wird hierbei zusammen mit dem Verabreichungssystem der WCL verwendet. Die Zusammenarbeit beruht auf früheren, von Cortecs durchgeführten klinischen Studien mit alpha-Interferon.

Interferone sind natürlich vorkommende Proteine oder Eiweisse. 'Roferon'-A ist das von Roche mittels Gentechnik produzierte Human-Interferon-alfa-2a; es ist in vielen Ländern als Mittel zur Behandlung von Haarzell-Leukämie, des durch AIDS verursachten Kaposi-Sarkoms und bösartiger Melanome zugelassen. Als weitere Indikationen werden derzeit registriert: chronische Hepatitis-B, kutanes T-Zell-Lymphom, chronisch-myeloische Leukämie und Nierenzellkrebs. Ausserdem wird 'Roferon'-A bei zusätzlichen Indikationen als Einzelsubstanz oder in Verbindung mit anderen Substanzen klinisch geprüft, so z. B. chronische Hepatitis-C, Darmkrebs sowie Lungenkrebs. Die Entwicklung einer oralen Form des Präparates könnte dic Behandlung dieser Krankheiten verbessern und den Alpha-Interferon-Markt erweitern.

In einer davon unabhängigen, zusätzlichen Entwicklung haben WCL und Cortecs im Mai 1990 bereits eine orale Form von Erythropoietin, dem kolonienstimulierenden Faktor roter Blutzellen, erfolgreich geprüft. Andere therapeutische Proteine, welche mit dem für grosse Moleküle bestimmten oralen Verabreichungssystem der WCL-Gruppe erfolgreich getestet wurden, schliessen Insulin, Calcitonin sowie menschliches Wachstumshormon ein.

\section{Fourth European Symposium on Radiopharmacy and Radiopharmaceuticals}

Baden (near Zürich), Switzerland Mai 1-4, 1991

Organized by the Radiopharmacy Group of the Swiss Society of Nuclear Medicine under the auspices of the EANM Taskgroup on Radiopharmaceuticals (Chairman: Prof. Dr. P. H. Cox)

The Fourth European Symposium will review recent developments in radiopharmacy and current research on radiopharmaceuticals. The scope will be expanded to include the pharmacokinetics and metabolism of radiopharmaceuticals, and the use of radioactive materials in drug formulation studies.

Secretary: c/o Radiopharmacy Division, Paul Scherrer Institutc, CH-5232 Villigen PSI, Switzerland, Telephone 0569928 14, Telex 827418 (psir ch), Telefax 056 982635 .

\section{EURO FOOD TOX III}

Schweizerische Chemische Gesellschaft Schweizerischer Chemiker-Verband

\section{Herbstversammlung in Bern}

Freitag, 19. Oktober 1990

Chemische Institute der Universität Bern, Freiestrasse 3

Programm $08.30-09.00$

09.00-09.30 Plenarvortrag des Werner-Preisträgers 1990 Dr. H. Frei, University of California Berkeley/USA

'Chemistry with red and near infrared light'

09.40-18.00 Kurzvorträge der Sektion für Organische Chemie

09.40-17.45 Vorträge der Sektion für Medizinische Chemie
09.40-15.40 Kurzvorträge der Sektion für Physikalische Chemie

14.20-17.00 Kurzvorträge der Sektion für Computerunterstützte Chemie

09.00-12.30 Minisymposium 'Recent Developments in Organometallic Chemistry and Homogeneous Catalysis'

14.30-16.30 Postersession der Sektion für Anorganische Chemie und Koordinationschemic

10.30-17.40 Seminar 'Analytische Chemie' 13.30-15.00 Postersession 'Analytische Chemie'

Information/Kontakt:

Dr. E. Zass, Sekretär SCG

ETH Zürich, Universitätstr. 16, CH-8092 Zürich
Interdisciplinary Conference on the Effects of Food on the Immune and Hormonal Systems sponsored by The European Society of Toxicology, The Federation of European Chemical Societies: Working Party on Food Chemistry, The Federation of European Nutrition Societies.

May 23-24, 1991, Zurich/Switzerland

Information: EURO FOOD TOX III, Sekretarial, MGB Zentrallabor, Postfach 266, 8031 Zürich, Switzerland.

\section{Der Schweizerische Schulrat}

hat den Ruzicka-Preis 1990 an Dr. Charles Fehr, Firmenich SA, Research Laboratories, Genf, verliehen. Anlässlich der Preisverleihung vom 10. September 1990 an der ETH Zürich hat der Preisträger folgenden Vortrag gehalten: Enantioselektive Protonierung von Enolaten zur Synthese von Naturstoffen. 
band für Umwelttechnik und dem Schweizerischen Elektrotechnischen Komitee (CES) Fachkommission 31 realisiert. Gezeigt werden neue Produkte und Technologien für den Einsatz elektrischer Betriebsmittel in explosionsgefährdeten Bereichen. Während der Dauer der Sonderschau stehen den Besuchern Mitglieder des Eidg. Starkstrominspektorates, des SEV (Schweizerischer Elektrotechnischer Verein), der SUVA und der Fachgruppe 31 für Fachgespräche und Auskünfte zur Verfügung.

Ein Hauptanliegen der Messe ist das Aufzeigen neuer Möglichkeiten und verbesserter Technologien zum Schutz von Mensch und Umwelt. In diese Richtung zielt nicht nur die Sonderschau. Das Thema der Messe verweist generell darauf, schaffen doch neue Werkstoffe wie auch neue Technologien die Grundlage für verfeinerte Methoden der Messung und der Kontrolle zum Schutz des Arbeitsplatzes und der Umwelt in der Produktion. Die ILMAC nimmt damit eine Herausforderung auf, welche mit der Diskussion um die Verantwortung der chemischen Industrie und der $\mathrm{Be}$ hörden verbunden ist.

Am begleitenden Kongress - dem 9. Basler Treffen für chemische Technik - finden zahlreiche Fachverbände den Rahmen zur Durchführung von Tagungen Wissenschaftler und Techniker aus aller Welt werden hier über den aktuellen Stand von Technik und Forschung berichten und diskutieren. Dem Besucher der ILMAC wird damit eine Bereicherung des Messebesuchs geboten.

\section{Vertreter der Schweiz wird Präsident des} europäischen Pharmaverbandes (EFPIA)

1.C. - Dr. Armin Kessler, Verwaltungsrat und Konzernleitungsmitglied der F. Hoffmann-La Roche AG, Basel, wurde anlässlich der EFPIA-Generalversammlung in Heidelberg einstimmig zum neuen Präsidenten für die Amtszeit 1990-92 gewählt. Kessler folgt auf $\mathrm{Dr}$ Alberto Aleotti (Menarini SpA) in einer Phase, die im Blick auf die Vollendung des EG-Binnenmarktes und die EWR-Verhandlungen für die pharmazeutische Industrie Europas von besonderer Bedeutung sein wird. Die EFPIA mit Sitz in Brüssel ist der europäische Pharmaverband, dem auch die Schweizerische Gesellschaft für Chemische Industrie (SGCI) als Vollmitglied angehört.

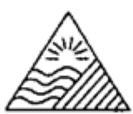

Internationa: Association of Environ mentel Analytical Chemistry Unter the patronage of the Swiss Feder

\section{2nd Soil Residue Analysis Workshop}

March 11-13, 1991

Ecole Polytechnique Fédérale de Lausanne (EPFL), Switzerland

Organization and information: Registration and Scientific Programme, Prof. Dr. Joseph Tarradellas, Chairman, IGE-EPFL, CH-1015 Lausanne, tel. (21) 693.27.12, fax. (21) 693.27.27
This Workshop is organized by the International Association of Environmental Analytical Chemistry and the Deparment of Analyticel Inorganic and Applied Chemistry of the University of Geneva. Swizertiand

Unter the Patronage of the Swiss Federal Otfice for the Environment, Forest and Landscape, Berne

\section{4th Hans Wolfgang Nürnberg \\ Memorial Workshop}

Toxic Metal Compounds

(Interrelation between Chemistry and Biology)

Congress-Center, CH-1865 Les Diablerets (Switzerland), March 4-8, 199

Organization and Information: Secretary and Address before and after the Workshop: Dr. Ernest Merian, Im Kirsgarten 22, CH-4106 Therwil (Switzerland), Tel. .. 4!-61-732950 (after 22nd April 1991 . 41-61-721 2950)

\section{Personalia}

\section{Geburtstage}

Alfred Hofstetter, Dr. sc. techn., Zürich, Mitglied des SChV, feiert am 3.10.90 seinen 60. Geburtstag.

Henri C. Silberman, Dr. chem., Laufen, Mitglied des SChV, feiert am 5.10 .90 seinen 65 . Geburtstag.

Rolf Schmid, Dr. Ing. Chem., Schwarzenburg, Mitglied des SChV, feiert am 6.10.90 seinen 65. Geburtstag.

Oswald Benz, Chemiker HTL, Grafenried, Mitglied des SChV, feiert am 11.10 .90 seinen 60 . Geburtstag.

Walter Frey, Dr. Ing. Chem., Muttenz, Mitglied des SChV, feiert am 12.10.90 seinen 75. Geburtstag.

Werner Manz, Dr. phil. II, Basel, Mitglied des SChV, feiert am 17.10.90 seinen 70. Geburtstag.

Ernst Kägi, Chemiker HTL, Monthey, Mitglied des SChV, feiert am 18.10 .90 seinen 60 . Geburtstag.

Max Sahli, Dr. phil., Chemiker, Kehrsatz, Mitglied des SChV, feiert am 23.10.90 seinen 65. Geburtstag.

Peter Jakober, Dr. sc. nat., Burgdorf, Mitglied des SChV, feiert am 24.10.90 seinen 60. Geburtstag.

Richard Neidlein, Prof. Dr., Heidelberg, Mitglied des SChV, feiert am 25.10 .90 seinen 60. Geburtstag.

U. Waltersperger, Chemiker HTL, Gossau, Mitglied des SChV, feiert am 29.10.90 seinen 65. Geburtstag.

\section{Neue Mitglieder}

Vogt-Schild AG, Druck und Verlag, Zuchwilerstrasse 21, 4501 Solothurn.

\section{Zusammenarbeit zwischen Roche und Western Capital für eine orale Form von Alpha-Interferon}

F. Hoffmann-La Roche AG, Basel, und die Western Capital Group (WCL) mit ihrer englischen PharmaTochtergesellschaft Cortecs Ltd. in London haben ein Abkommen über dic Zusammenarbeit zur Entwicklung und Prüfung einer oralen Form von alpha-Interferon unterzeichnet. Das Roche Präparat 'Roferon'-A wird hierbei zusammen mit dem Verabreichungssystem der WCL verwendet. Die Zusammenarbeit beruht auf früheren, von Cortecs durchgeführten klinischen Studien mit alpha-Interferon.

Interferone sind natürlich vorkommende Proteine oder Eiweisse. 'Roferon'-A ist das von Roche mittels Gentechnik produzierte Human-Interferon-alfa-2a; es ist in vielen Ländern als Mittel zur Behandlung von Haarzell-Leukämie, des durch AIDS verursachten Kaposi-Sarkoms und bösartiger Melanome zugelassen. Als weitere Indikationen werden derzeit registriert: chronische Hepatitis-B, kutanes T-Zell-Lymphom, chronisch-myeloische Leukämie und Nierenzellkrebs. Ausserdem wird 'Roferon'-A bei zusätzlichen Indikationen als Einzelsubstanz oder in Verbindung mit anderen Substanzen klinisch geprüft, so z. B. chronische Hepatitis-C, Darmkrebs sowie Lungenkrebs. Die Entwicklung einer oralen Form des Präparates könnte dic Behandlung dieser Krankheiten verbessern und den Alpha-Interferon-Markt erweitern.

In einer davon unabhängigen, zusätzlichen Entwicklung haben WCL und Cortecs im Mai 1990 bereits eine orale Form von Erythropoietin, dem kolonienstimulierenden Faktor roter Blutzellen, erfolgreich geprüft. Andere therapeutische Proteine, welche mit dem für grosse Moleküle bestimmten oralen Verabreichungssystem der WCL-Gruppe erfolgreich getestet wurden, schliessen Insulin, Calcitonin sowie menschliches Wachstumshormon ein.

\section{Fourth European Symposium on Radiopharmacy and Radiopharmaceuticals}

Baden (near Zürich), Switzerland Mai 1-4, 1991

Organized by the Radiopharmacy Group of the Swiss Society of Nuclear Medicine under the auspices of the EANM Taskgroup on Radiopharmaceuticals (Chairman: Prof. Dr. P. H. Cox)

The Fourth European Symposium will review recent developments in radiopharmacy and current research on radiopharmaceuticals. The scope will be expanded to include the pharmacokinetics and metabolism of radiopharmaceuticals, and the use of radioactive materials in drug formulation studies.

Secretary: c/o Radiopharmacy Division, Paul Scherrer Institutc, CH-5232 Villigen PSI, Switzerland, Telephone 0569928 14, Telex 827418 (psir ch), Telefax 056 982635 .

\section{EURO FOOD TOX III}

Schweizerische Chemische Gesellschaft Schweizerischer Chemiker-Verband

\section{Herbstversammlung in Bern}

Freitag, 19. Oktober 1990

Chemische Institute der Universität Bern, Freiestrasse 3

Programm $08.30-09.00$

09.00-09.30 Plenarvortrag des Werner-Preisträgers 1990 Dr. H. Frei, University of California Berkeley/USA

'Chemistry with red and near infrared light'

09.40-18.00 Kurzvorträge der Sektion für Organische Chemie

09.40-17.45 Vorträge der Sektion für Medizinische Chemie
09.40-15.40 Kurzvorträge der Sektion für Physikalische Chemie

14.20-17.00 Kurzvorträge der Sektion für Computerunterstützte Chemie

09.00-12.30 Minisymposium 'Recent Developments in Organometallic Chemistry and Homogeneous Catalysis'

14.30-16.30 Postersession der Sektion für Anorganische Chemie und Koordinationschemic

10.30-17.40 Seminar 'Analytische Chemie' 13.30-15.00 Postersession 'Analytische Chemie'

Information/Kontakt:

Dr. E. Zass, Sekretär SCG

ETH Zürich, Universitätstr. 16, CH-8092 Zürich
Interdisciplinary Conference on the Effects of Food on the Immune and Hormonal Systems sponsored by The European Society of Toxicology, The Federation of European Chemical Societies: Working Party on Food Chemistry, The Federation of European Nutrition Societies.

May 23-24, 1991, Zurich/Switzerland

Information: EURO FOOD TOX III, Sekretarial, MGB Zentrallabor, Postfach 266, 8031 Zürich, Switzerland.

\section{Der Schweizerische Schulrat}

hat den Ruzicka-Preis 1990 an Dr. Charles Fehr, Firmenich SA, Research Laboratories, Genf, verliehen. Anlässlich der Preisverleihung vom 10. September 1990 an der ETH Zürich hat der Preisträger folgenden Vortrag gehalten: Enantioselektive Protonierung von Enolaten zur Synthese von Naturstoffen. 